\title{
The integral moments and ratios of quadratic Dirichlet $L$-functions over monic irreducible polynomials in $\mathbb{F}_{q}[T]$
}

\author{
J. C. Andrade ${ }^{1} \oplus \cdot$ H. Jung ${ }^{2} \cdot$ A. Shamesaldeen ${ }^{1}$
}

Received: 15 July 2019 / Accepted: 1 March 2021 / Published online: 5 May 2021

(c) The Author(s) 2021

\begin{abstract}
In this paper, we extend to the function field setting the heuristics formerly developed by Conrey, Farmer, Keating, Rubinstein and Snaith, for the integral moments of $L$ functions. We also adapt to the function field setting the heuristics first developed by Conrey, Farmer and Zirnbauer to the study of mean values of ratios of $L$-functions. Specifically, the focus of this paper is on the family of quadratic Dirichlet $L$-functions $L\left(s, \chi_{P}\right)$ where the character $\chi$ is defined by the Legendre symbol for polynomials in $\mathbb{F}_{q}[T]$ with $\mathbb{F}_{q}$ a finite field of odd cardinality, and the averages are taken over all monic and irreducible polynomials $P$ of a given odd degree. As an application, we also compute the formula for the one-level density for the zeros of these $L$-functions.
\end{abstract}

Keywords Function fields · Integral moments of $L$-functions · Quadratic Dirichlet $L$-functions · Ratios conjecture

Mathematics Subject Classification Primary 11M38; Secondary 11M06 · 11G20 . $11 \mathrm{M} 50 \cdot 14 \mathrm{G} 10$

\begin{abstract}
The first author is grateful to the Leverhulme Trust (Grant No. RPG-2017-320) for the support through the research project grant "Moments of L-functions in Function Fields and Random Matrix Theory". The research of the second author is supported by the National Research Foundation of Korea (NRF) grant funded by the Korea government (MSIT) (2020R1F1A1A01066105). The third author is supported by a Ph.D. scholarship from the government of Kuwait.
\end{abstract}

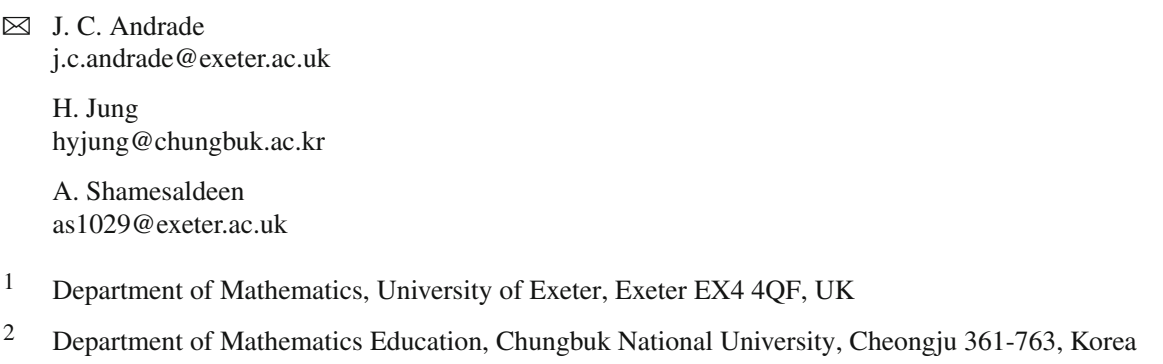




\section{Introduction}

In this paper, we study the family of quadratic Dirichlet $L$-functions $L\left(s, \chi_{P}\right)$ where the character $\chi$ is defined by the Legendre symbol $(\dot{\bar{P}})$ and $P$ ranges over monic irreducible polynomials of degree $2 g+1$ over $\mathbb{F}_{q}[T]$. We present the conjectures for the moments and ratios of this family of $L$-functions, Conjectures 2.2 and 2.3, respectively, by making use of the recipe developed by Conrey et al. in [7] and adapting it for this family of $L$-functions.

The study of moments of families of $L$-functions is a central topic in analytic number theory. Many mathematicians have studied this subject, and considerable progress was made in the last decades in the direction of getting a better understanding of the asymptotic behaviour of such moments. For example, in the case of the Riemann zeta-function, the problem is to understand the asymptotic behaviour of

$$
M_{k}(T)=\int_{0}^{T}\left|\zeta\left(\frac{1}{2}+i t\right)\right|^{2 k} \mathrm{~d} t
$$

as $T \rightarrow \infty$.

Hardy and Littlewood [22] proved in 1918 an asymptotic formula for the second moment, i.e.

$$
M_{1}(T) \sim T \log T
$$

In 1926, Ingham [24] showed that when $k=2$,

$$
M_{2}(T) \sim \frac{1}{2 \pi^{2}} T(\log T)^{4}
$$

For values of $k \geq 3$, it still remains an unsolved problem to obtain asymptotic formulas for $M_{k}(T)$. However, it is conjectured that for every $k \geq 0$, there is a constant $c_{k}$ such that

$$
M_{k}(T) \sim c_{k} T(\log T)^{k^{2}} .
$$

Conrey and Ghosh [13] made a conjecture for the sixth moment of the Riemann zeta-function, and later on, Conrey and Gonek [14] put forward a conjecture for the eighth moment but their approach fails to provide conjectures for higher moments. Keating and Snaith [28], using random matrix theory, conjectured the precise value of the constant $c_{k}$ for all values of $k>0$. In fact, their conjecture produces a value for $c_{k}$ for $\mathfrak{R}(k)>-1 / 2$. More recently, Conrey and Keating, in a series of papers [9-12], returned to the problem of obtaining conjectures for the higher moments of the Riemann zeta-function using only number-theoretic heuristics. Their new approach produced conjectures for moments of the Riemann zeta function, as well as explained the role of the non-diagonal contribution to the main terms in the asymptotic formulas.

A different example is the family of quadratic Dirichlet $L$-functions $L\left(s, \chi_{d}\right)$, where $\chi_{d}$ is the real primitive Dirichlet character modulo $d$ defined by the Kronecker symbol $\chi_{d}(n)=\left(\frac{d}{n}\right)$. The problem here is to establish an asymptotic formula for 


$$
\sum_{d \leq X} L\left(\frac{1}{2}, \chi_{d}\right)^{k}
$$

as $X \rightarrow \infty$, where the sum is taken over all positive discriminants $d$ and $k$ is a positive integer. In this case, as it is for the Riemann zeta-function, just the first few moments were computed. In 1981, Jutila [25] established the asymptotic formula for the first and second moments. The asymptotic formulas he obtained are

$$
\sum_{d \leq X} L\left(\frac{1}{2}, \chi_{d}\right) \sim C_{1} X \log X
$$

and

$$
\sum_{d \leq X} L\left(\frac{1}{2}, \chi_{d}\right)^{2} \sim C_{2} X(\log X)^{3},
$$

where the constants $C_{1}$ and $C_{2}$ can be expressed in terms of Euler products and factors containing the Riemann zeta-function. Soundararajan [32] computed the asymptotic formula for the third moment. He proved that

$$
\sum_{d \leq X} L\left(\frac{1}{2}, \chi_{8 d}\right)^{3} \sim C_{3} X(\log X)^{6}
$$

where $d$ is an odd, square-free and positive number, $\chi_{8 d}$ is a real, even primitive Dirichlet character with conductor $8 d$, and $C_{3}$ is a constant.

In another paper, Soundararajan and Young [33] claimed that they are able to establish an asymptotic formula for the fourth power moment for this family of $L$ functions under the Generalised Riemann Hypothesis (GRH). The claim is that

$$
\sum_{d \leq X} L\left(\frac{1}{2}, \chi_{d}\right)^{4} \sim C_{4} X(\log X)^{10}
$$

where $C_{4}$ is constant. Recently, Shen [31] proved the asymptotic formula for the fourth moment of quadratic Dirichlet $L$-functions under the Generalised Riemann Hypothesis $(\mathrm{GRH}) . \mathrm{He}$ consider the characters of the form $\chi_{8 d}$ and has established that

$$
\sum_{\substack{d \leq X \\(d, 2)=1}}^{*} L\left(\frac{1}{2}, \chi_{8 d}\right)^{4} \sim \frac{a_{4}}{2^{6} \cdot 3^{3} \cdot 5^{2} \cdot 7 \cdot \pi} X(\log X)^{10},
$$

where $a_{4}$ is as defined in [27].

In 2005, Conrey et al. [7] presented a new heuristic for all of the main terms in the integral moments of several families of primitive $L$-functions. Their conjectures agree with previously known results. For the Riemann zeta-function, they gave a precise conjecture for $M_{k}(T)$ including an asymptotic expansion for the lower-order terms using shifted moments. For the family of quadratic Dirichlet $L$-functions, they conjectured that 


$$
\sum_{d} L\left(\frac{1}{2}, \chi_{d}\right)^{k}=\sum_{d} Q_{k}(\log |d|)(1+o(1))
$$

where $Q_{k}$ is polynomial of degree $k(k+1) / 2$ with $k \in \mathbb{N}$.

It is important to observe that Diaconu et al. [16] have also conjectured moments of families of $L$-functions using different techniques. Their method is based on multiple Dirichlet series. Recently, Diaconu and Whitehead [17] established a smoothed asymptotic formula for the third moment of quadratic Dirichlet $L$-functions at the central value. In addition to the main term, which is known, they prove the existence of a secondary term of size $x^{3 / 4}$. The error term in their asymptotic formula is on the order of $O\left(x^{2 / 3+\delta}\right)$ for every $\delta>0$.

In 2008, Conrey et al. [8] presented a generalisation of the heuristic method for moments presented in [7] to the case of ratios of products of $L$-functions. These conjectures are very powerful since they encode information about statistics of zeros of such $L$-functions. The ratios conjectures as put forward by Conrey, Farmer and Zirnbauer can be used to prove very precise results about the distribution of zeros of families of $L$-functions such as pair-correlation and $n$-level density (for more details see [15]). Their ratios conjecture for the family of quadratic Dirichlet $L$-functions are read as follow.

Conjecture 1.1 (Conrey, Farmer, Zirnbauer) Let $\mathcal{D}=\left\{L\left(s, \chi_{d}\right): d>0\right\}$ to be the symplectic family of $L$-functions associated with the quadratic character $\chi_{d}$. For positive real parts of $\alpha_{k}$ and $\gamma_{m}$, we have

$$
\begin{aligned}
& \sum_{0<d \leq X} \frac{\prod_{k=1}^{K} L\left(\frac{1}{2}+\alpha_{k}, \chi_{d}\right)}{\prod_{m=1}^{Q} L\left(\frac{1}{2}+\gamma_{m}, \chi_{d}\right)} \\
& =\sum_{0<d \leq X} \sum_{\varepsilon \in\{-1,1\}^{K}}\left(\frac{|d|}{\pi}\right)^{\frac{1}{2} \sum_{k=1}^{K}\left(\varepsilon_{k} \alpha_{k}-\alpha_{k}\right)} \\
& \quad \times \prod_{k=1}^{K} g_{+}\left(\frac{1}{2}+\frac{\alpha_{k}-\varepsilon_{k} \alpha_{k}}{2}\right) Y\left(\varepsilon_{1} \alpha_{1}, \ldots, \varepsilon_{K} \alpha_{K} ; \gamma\right) A_{\mathcal{D}}\left(\varepsilon_{1} \alpha_{1}, \ldots, \varepsilon_{K} \alpha_{K} ; \gamma\right) \\
& \quad+o(X),
\end{aligned}
$$

where

$$
\begin{aligned}
g_{+}(s) & =\frac{\Gamma\left(\frac{1-s}{2}\right)}{\Gamma\left(\frac{s}{2}\right)}, \\
Y(\alpha ; \gamma) & =\frac{\prod_{j \leq k \leq K} \zeta\left(1+\alpha_{j}+\alpha_{k}\right) \prod_{m \leq r \leq Q} \zeta\left(1+\gamma_{m}+\gamma_{r}\right)}{\prod_{k=1}^{K} \prod_{m=1}^{Q} \zeta\left(1+\alpha_{k}+\gamma_{m}\right)}
\end{aligned}
$$


and

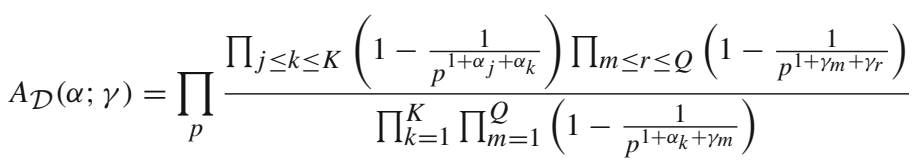

$$
\begin{aligned}
& \times\left(1+\left(1+\frac{1}{p}\right)^{-1} \sum_{0<\sum_{k} a_{k}+\sum_{m} c_{m} \text { is even }} \frac{\prod_{m=1}^{Q} \mu\left(P^{c_{m}}\right)}{\sum_{k} a_{k}\left(\frac{1}{2}+\alpha_{k}\right)+\sum_{m} c_{m}\left(\frac{1}{2}+\gamma_{m}\right)}\right) .
\end{aligned}
$$

In 1979, Goldfeld and Viola [21] introduced a variant of the problem about moments of quadratic Dirichlet $L$-functions. They conjectured an asymptotic formula for

$$
\sum_{\substack{p \leq X \\ p \equiv 3(\bmod 4)}} L\left(\frac{1}{2}, \chi_{p}\right)
$$

where the sum is taken over prime numbers and $\chi_{p}(n)=\left(\frac{n}{p}\right)$ is the usual Legendre symbol. In this direction, Jutila [25] studied the first moment of this family of $L$ functions and proved that

$$
\sum_{\substack{p \leq X \\ p \equiv 3(\bmod 4)}}(\log p) L\left(\frac{1}{2}, \chi_{p}\right) \sim \frac{1}{4} X \log X
$$

Recently, assuming the Generalised Riemann Hypothesis (GRH), Baluyot and Pratt [5] obtained the leading order term for the second moment. They proved that

$$
\sum_{\substack{p \leq X \\ p \equiv 1(\bmod 8)}}(\log p) L\left(\frac{1}{2}, \chi_{p}\right)^{2}=c \frac{X}{4}(\log X)^{3}+O\left(X(\log X)^{11 / 4}\right),
$$

where $c$ is a positive constant.

We should notice that the second moment for this family of $L$-functions seems to be the limit of the current technology. This is in part due to the fact that for this family, we are dealing with character sums over prime numbers, and these sums are more complicated than those over square-free numbers. For example, in the case for square-free numbers, it was possible to obtain the third moment by making use of the Poisson summation formula, but the same does not seem to apply for the family over prime numbers since we cannot directly apply Poisson to the sums over primes.

\subsection{The function field setting}

Let $\mathcal{H}_{2 g+1, q}$ be the hyperelliptic ensemble of monic, square-free polynomials of degree $2 g+1$. When the cardinality of the field $\mathbb{F}_{q}$ is $q \equiv 1(\bmod 4)$, Andrade and Keating [2] computed the first moment of the family of $L$-functions associated to the quadratic character $\chi_{D}$, with $D \in \mathcal{H}_{2 g+1, q}$. They proved that 


$$
\sum_{D \in \mathcal{H}_{2 g+1, q}} L\left(\frac{1}{2}, \chi_{D}\right) \sim|D| P_{1}\left(\log _{q}|D|\right)
$$

where $P_{1}$ is a linear polynomial. For the second, third and fourth moments of this family, Florea $[18,19]$ proved that

$$
\sum_{D \in \mathcal{H}_{2 g+1, q}} L\left(\frac{1}{2}, \chi_{D}\right)^{k} \sim|D| P_{k}\left(\log _{q}|D|\right)
$$

where $P_{k}$ is a polynomial of degree 3, 6 and 10 respectively, whose coefficients can be computed explicitly, except for $P_{4}$ where only the first few coefficients were obtained. It is worth noticing that Florea in [20] improved the error term for the first moment and was able to obtain a strenuous lower-order term that was never predicted by random matrix theory or other heuristics.

In another paper, Andrade and Keating [4] adapted the recipe of [7] and of [8] to the function field setting and conjectured asymptotic formulas for the integral moments and ratios of the family of quadratic Dirichlet $L$-functions in function fields. Their main conjectures are presented below.

Conjecture 1.2 (Andrade and Keating-Integral Moments Conjecture) Suppose that $q$ odd is the fixed cardinality of the finite field $\mathbb{F}_{q}$ and let $\mathcal{X}_{D}(s)=|D|^{1 / 2-s} \mathcal{X}(s)$ and

$$
\mathcal{X}(s)=q^{-1 / 2+s} .
$$

That is $\mathcal{X}_{D}(s)$ is the factor in the functional equation

$$
L\left(s, \chi_{D}\right)=\mathcal{X}_{D}(s) L\left(1-s, \chi_{D}\right)
$$

Summing over fundamental discriminants $D \in \mathcal{H}_{2 g+1, q}$, we have

$$
\sum_{D \in \mathcal{H}_{2 g+1, q}} L\left(\frac{1}{2}, \chi_{D}\right)^{k}=\sum_{D \in \mathcal{H}_{2 g+1, q}} Q_{k}\left(\log _{q}|D|\right)(1+o(1)),
$$

where $Q_{k}$ is polynomial of degree $k(k+1) / 2$ given by the $k$-fold residue

$$
\begin{aligned}
Q_{k}(x)= & \frac{(-1)^{k(k-1) / 2} 2^{k}}{k !} \frac{1}{(2 \pi i)^{k}} \oint \cdots \oint \frac{G\left(z_{1}, \ldots, z_{k}\right) \triangle\left(z_{1}^{2}, \ldots, z_{k}^{2}\right)^{2}}{\prod_{i=1}^{k} z_{i}^{2 k-1}} \\
& \times q^{\frac{x}{2} \sum_{i=1}^{k} z_{i}} \mathrm{~d} z_{1} \ldots z_{k},
\end{aligned}
$$

$\Delta\left(z_{1}, \ldots, z_{k}\right)$ the Vandermonde determinant given by

$$
\begin{aligned}
& \Delta\left(z_{1}, \ldots, z_{k}\right)=\prod 1 \leq i \leq j \leq k\left(z_{j}-z_{i}\right) \\
& G\left(z_{1}, \ldots, z_{k}\right)=A_{k}\left(\frac{1}{2} ; z_{1}, \ldots, z_{k}\right) \prod_{i=1}^{k} \mathcal{X}\left(\frac{1}{2}+z_{i}\right)^{-\frac{1}{2}} \prod_{1 \leq i \leq j \leq k} \zeta_{A}\left(1+z_{i}+z_{j}\right),
\end{aligned}
$$


and $A_{k}$ is the Euler product, absolutely convergent for $\left|\Re\left(z_{i}\right)\right|<\frac{1}{2}$, defined by

$$
\begin{aligned}
A_{k}\left(\frac{1}{2} ; z_{1}, \ldots, z_{k}\right)= & \prod_{\substack{P \text { monic } \\
\text { irreducible }}} \prod_{1 \leq i \leq j \leq k}\left(1-\frac{1}{|P|^{1+z_{i}+z_{j}}}\right) \\
& \times\left(\frac{1}{2}\left(\prod_{i=1}^{k}\left(1-\frac{1}{|P|^{\frac{1}{2}+z_{i}}}\right)^{-1}+\prod_{i=1}^{k}\left(1+\frac{1}{|P|^{\frac{1}{2}+z_{i}}}\right)^{-1}\right)+\frac{1}{|P|}\right) \\
& \times\left(1+\frac{1}{|P|}\right)^{-1}
\end{aligned}
$$

Conjecture 1.3 (Andrade and Keating-Ratios Conjecture) Let $\alpha_{k}$ and $\gamma_{m}$ complex numbers with positive and small real parts. Let $\mathfrak{D}=\left\{L\left(s, \chi_{D}\right): D \in \mathcal{H}_{2 g+1, q}\right\}$ to be the family of $L$-functions associated with the quadratic character $\chi_{D}$. Then,

$$
\begin{aligned}
& \sum_{D \in \mathcal{H}_{2 g+1, q}} \frac{\prod_{k=1}^{K} L\left(\frac{1}{2}+\alpha_{k}, \chi_{D}\right)}{\prod_{m=1}^{Q} L\left(\frac{1}{2}+\gamma_{m}, \chi_{D}\right)} \\
& =\sum_{D \in \mathcal{H}_{2 g+1, q}} \sum_{\varepsilon \in\{-1,1\}^{k}}|D|^{-\frac{1}{2} \sum_{k=1}^{K}\left(\varepsilon_{k} \alpha_{k}-\alpha_{k}\right)} \prod_{k=1}^{K} \mathcal{X}\left(\frac{1}{2}+\frac{\alpha_{k}-\varepsilon_{k} \alpha_{k}}{2}\right) \\
& \quad \times Y_{\mathfrak{D}}\left(\varepsilon_{1} \alpha_{1}, \ldots, \varepsilon_{K} \alpha_{K} ; \gamma\right) A_{\mathfrak{D}}\left(\varepsilon_{1} \alpha_{1}, \ldots, \varepsilon_{K} \alpha_{K} ; \gamma\right)+o(D),
\end{aligned}
$$

with

$A_{\mathfrak{D}}(\alpha ; \gamma)$

$$
\begin{aligned}
= & \prod_{\substack{P \text { monic } \\
\text { irreducible }}} \frac{\prod_{j \leq k \leq K}\left(1-\frac{1}{|P|^{1+\alpha_{j}+\alpha_{k}}}\right) \prod_{m \leq r \leq Q}\left(1-\frac{1}{|P|^{1+\gamma_{m}+\gamma_{r}}}\right)}{\prod_{k=1}^{K} \prod_{m=1}^{Q}\left(1-\frac{1}{|P|^{1+\alpha_{k}+\gamma_{m}}}\right)} \\
& \times\left(1+\left(1+\frac{1}{|P|}\right)^{-1} \sum_{0<\sum_{k} a_{k}+\sum_{m} c_{m} \text { is even }} \frac{\prod_{m=1}^{Q} \mu\left(P^{c_{m}}\right)}{|P|^{\sum_{k} a_{k}\left(\frac{1}{2}+\alpha_{k}\right)+\sum_{m} c_{m}\left(\frac{1}{2}+\gamma_{m}\right)}}\right)
\end{aligned}
$$

and

$$
Y_{\mathfrak{D}}(\alpha ; \gamma)=\frac{\prod_{j \leq k \leq K} \zeta_{A}\left(1+\alpha_{j}+\alpha_{k}\right) \prod_{m \leq r \leq Q} \zeta_{A}\left(1+\gamma_{m}+\gamma_{r}\right)}{\prod_{k=1}^{K} \prod_{m=1}^{Q} \zeta_{A}\left(1+\alpha_{k}+\gamma_{m}\right)},
$$

where $\zeta_{A}(s)$ is the zeta-function associated to the polynomial ring $A=\mathbb{F}_{q}[T]$ and $\mathcal{X}(s)$ is a function that depends on $q$.

One can note that (1.21) and (1.26) are the function field analogues of the formulas (1.11) and (1.12), respectively. 
The main aim of this paper is to formulate a conjectural asymptotic formula for

$$
\sum_{P \in \mathcal{P}_{2 g+1, q}} L\left(\frac{1}{2}, \chi_{P}\right)^{k}
$$

where $\mathcal{P}_{2 g+1, q}$ is the set of all monic, irreducible polynomials of odd degree $2 g+1$ with coefficients in $\mathbb{F}_{q}$, as $|P| \rightarrow \infty$.

Andrade and Keating [3] established asymptotic formulas for the first and second moments of (1.29), namely

$$
\begin{aligned}
& \sum_{P \in \mathcal{P}_{2 g+1, q}}\left(\log _{q}|P|\right) L\left(\frac{1}{2}, \chi_{P}\right) \sim \frac{1}{2}|P|\left(\log _{q}|P|+1\right), \\
& \sum_{P \in \mathcal{P}_{2 g+1, q}} L\left(\frac{1}{2}, \chi_{P}\right)^{2} \sim \frac{1}{24} \frac{1}{\zeta_{A}(2)}|P|\left(\log _{q}|P|\right)^{2}
\end{aligned}
$$

Recently, Bui and Florea [6] improved Andrade and Keating's result for the second moment and proved that

$$
\frac{1}{\left|\mathcal{P}_{2 g+1, q}\right|} \sum_{P \in \mathcal{P}_{2 g+1, q}} L\left(\frac{1}{2}, \chi_{P}\right)^{2}=\frac{g^{3}}{3 \zeta_{A}(2)}+g^{2}\left(\frac{3}{2}+\frac{1}{2 q}\right)+O_{\varepsilon}\left(g^{3 / 2+\varepsilon}\right) \text {. }
$$

In this paper, we adapt to the function field case the recipe for the conjectures of the moments and ratios of $L$-functions for the family of quadratic Dirichlet $L$-functions associated with $\chi_{P}$ over a fixed finite field $\mathbb{F}_{q}$. In Sect. 2 , we present some basic facts on $L$-functions over function fields followed by the statement of our main results. In Sect. 3, we present the details of the recipe of [7] when it is adapted for the function field setting. In Sect. 4, we use the integral moments conjecture over function fields when $k=1,2$, and compare with the main theorems of [4]. Then we conjecture the precise value for the third moment, i.e. when $k=3$ in this setting. In Sect. 5, we present the recipe of [8] for the same family of $L$-functions over function fields. In Sect. 6, we use the ratios conjecture for function fields and compute the one-level density of the zeros of this same family of $L$-functions.

\section{Statement of the main results}

In this section, we gather some basic facts about $L$-functions over function fields. Many of the results and notation here can also be found in [29].

Let $\mathbb{F}_{q}$ be a finite field of odd cardinality $q=p^{a}$, with $p$ a prime. Denote the polynomial ring over $\mathbb{F}_{q}$ by $A=\mathbb{F}_{q}[T]$, and the rational function field by $k=\mathbb{F}_{q}(T)$. For a polynomial $f$ in $\mathbb{F}_{q}[T]$, we define the norm of $f$ by $|f|:=q^{\operatorname{deg}(f)}$. For $\Re(s)>1$, the zeta-function attached to $A$ is defined by 


$$
\zeta_{A}(s)=\sum_{f \text { monic }} \frac{1}{|f|^{s}}=\prod_{\substack{P \text { monic } \\ \text { irreducible }}}\left(1-|P|^{-s}\right)^{-1}
$$

Since there are $q^{n}$ monic polynomials of degree $n$, we can easily prove that

$$
\zeta_{A}(s)=\frac{1}{1-q^{1-s}}
$$

which provides an analytic continuation of the zeta-function to the whole complex plane, with simple pole at $s=1$, which leads to the analogue of the Prime Number Theorem for polynomials in $A=\mathbb{F}_{q}[T]$.

Theorem 2.1 (Prime Polynomial Theorem) Let $\pi_{A}(n)$ denote the number of monic irreducible polynomials of degree $n$ in A. Then

$$
\pi_{A}(n)=\frac{q^{n}}{n}+O\left(\frac{q^{n / 2}}{n}\right) .
$$

Now, let $P$ be a monic irreducible polynomial, define the quadratic character $\left(\frac{f}{P}\right)$ by

$$
\left(\frac{f}{P}\right)= \begin{cases}1 & \text { if } f \text { is a square }(\bmod P), P \nmid f, \\ -1 & \text { if } f \text { is not a square }(\bmod P), P \nmid f, \\ 0 & \text { if } P \mid f .\end{cases}
$$

The quadratic reciprocity law states that for $A, B$ non-zeros and relatively prime monic polynomials, we have

$$
\left(\frac{A}{B}\right)=\left(\frac{B}{A}\right)(-1)^{\frac{q-1}{2} \operatorname{deg}(A) \operatorname{deg}(B)} .
$$

We denote by $\chi_{P}$ the quadratic character defined in terms of the quadratic residue symbol for $A$

$$
\chi_{P}(f)=\left(\frac{P}{f}\right)
$$

where $f \in A$.

In this paper, the focus will be in the family of quadratic Dirichlet $L$-functions associated with polynomials $P \in \mathcal{P}_{2 g+1, q}$, where

$$
\mathcal{P}_{2 g+1, q}=\{P \in A, \text { monic, irreducible and } \operatorname{deg}(P)=2 g+1\} .
$$


The quadratic Dirichlet $L$-function attached to the character $\chi_{P}$ is defined to be

$$
\begin{aligned}
L\left(s, \chi_{P}\right) & =\sum_{\substack{f \in A \\
f \text { monic }}} \frac{\chi_{P}(f)}{|f|^{s}} \\
& =\prod_{\substack{P \text { monic } \\
\text { irreducible }}}\left(1-\chi_{P}(P)|P|^{-s}\right)^{-1}, \quad \mathfrak{R}(s)>1 .
\end{aligned}
$$

With the change of variables $u=q^{-s}, L\left(s, \chi_{P}\right)$ is a polynomial of degree $2 g$ given by

$$
L\left(s, \chi_{P}\right)=\mathcal{L}\left(u, \chi_{P}\right)=\sum_{n=0}^{2 g} \sum_{\substack{f \text { monic } \\ \operatorname{deg}(f)=n}} \chi_{P}(f) u^{n} .
$$

(see Propositions 14.6 and 17.7 in [29]).

We are now in a position to state the main conjectures of this paper.

Conjecture 2.2 Suppose that $q \equiv 1(\bmod 4)$ is the fixed cardinality of the finite field $\mathbb{F}_{q}$ and let $\mathcal{X}_{P}(s)=|P|^{1 / 2-s} \mathcal{X}(s)$ where

$$
\mathcal{X}(s)=q^{-1 / 2+s} .
$$

That is $\mathcal{X}_{P}(s)$ is the factor in the functional equation

$$
L\left(s, \chi_{P}\right)=\mathcal{X}_{P}(s) L\left(1-s, \chi_{p}\right)
$$

Summing over primes $P \in \mathcal{P}_{2 g+1, q}$, we have

$$
\sum_{P \in \mathcal{P}_{2 g+1, q}} L\left(\frac{1}{2}, \chi_{P}\right)^{k}=\sum_{P \in \mathcal{P}_{2 g+1, q}} Q_{k}\left(\log _{q}|P|\right)(1+o(1))
$$

where $Q_{k}$ is polynomial of degree $k(k+1) / 2$ given by the $k$-fold residue

$$
\begin{aligned}
Q_{k}(x)= & \frac{(-1)^{k(k-1) / 2} 2^{k}}{k !} \frac{1}{(2 \pi i)^{k}} \oint \ldots \oint \frac{G\left(z_{1}, \ldots, z_{k}\right) \Delta\left(z_{1}^{2}, \ldots, z_{k}^{2}\right)^{2}}{\prod_{i=1}^{k} z_{i}^{2 k-1}}, \\
& \times q^{\frac{x}{2} \sum_{i=1}^{k} z_{i}} \mathrm{~d} z_{1} \ldots z_{k}
\end{aligned}
$$

where $\Delta\left(z_{1}, \ldots, z_{k}\right)$ is defined as in (1.23),

$$
G\left(z_{1}, \ldots, z_{k}\right)=A_{k}\left(\frac{1}{2} ; z_{1}, \ldots, z_{k}\right) \prod_{i=1}^{k} \mathcal{X}\left(\frac{1}{2}+z_{i}\right)^{-\frac{1}{2}} \prod_{1 \leq i \leq j \leq k} \zeta_{A}\left(1+z_{i}+z_{j}\right)
$$


and $A_{k}$ is the Euler product, absolutely convergent for $\left|\Re\left(z_{i}\right)\right|<\frac{1}{2}$, defined by

$$
\begin{aligned}
A_{k}\left(\frac{1}{2} ; z_{1}, \ldots, z_{k}\right)= & \prod_{\substack{P \text { monic } \\
\text { irreducible }}} \prod_{1 \leq i \leq j \leq k}\left(1-\frac{1}{|P|^{1+z_{i}+z_{j}}}\right) \\
& \times \frac{1}{2}\left(\prod_{i=1}^{k}\left(1-\frac{1}{|P|^{\frac{1}{2}+z_{i}}}\right)^{-1}+\prod_{i=1}^{k}\left(1+\frac{1}{|P|^{\frac{1}{2}+z_{i}}}\right)^{-1}\right) .
\end{aligned}
$$

More generally, we have

$$
\begin{aligned}
\sum_{P \in \mathcal{P}_{2 g+1, q}} L & \left(\frac{1}{2}+\alpha_{1}, \chi_{P}\right) \cdots L\left(\frac{1}{2}+\alpha_{k}, \chi_{P}\right) \\
\quad= & \sum_{P \in \mathcal{P}_{2 g+1, q}} \prod_{i=1}^{k} \mathcal{X}\left(\frac{1}{2}+\alpha_{i}\right)^{-\frac{1}{2}}|P|^{-\frac{1}{2} \sum_{i=1}^{k} \alpha_{i}} Q_{k}\left(\log _{q}|P|, \alpha\right)(1+o(1))
\end{aligned}
$$

where

$$
\begin{aligned}
Q_{k}(x, \alpha)= & \frac{(-1)^{k(k-1) / 2} 2^{k}}{k !} \frac{1}{(2 \pi i)^{k}} \oint \cdots \oint \frac{G\left(z_{1}, \cdots, z_{k}\right) \Delta\left(z_{1}^{2}, \ldots, z_{k}^{2}\right)^{2} \prod_{i=1}^{k} z_{i}}{\prod_{i=1}^{k} \prod_{j=1}^{k}\left(z_{j}-\alpha_{i}\right)\left(z_{j}+\alpha_{i}\right)} \\
& \times q^{\frac{x}{2} \sum_{i=1}^{k} z_{i}} \mathrm{~d} z_{1} \cdots z_{k},
\end{aligned}
$$

and the path of integration encloses the $\pm \alpha$ 's.

Note that, for the cases $k=1,2$, our conjecture agrees with Andrade and Keating's results in (1.30) and (1.31) and Bui and Florea's result in (1.32). See Sects. 4.1 and 4.2 for further details.

The next conjecture is the translation for function fields of the ratios conjecture for quadratic Dirichlet $L$-functions associated with the characters $\chi_{P}$.

Conjecture 2.3 Suppose that the real parts of $\alpha_{k}$ and $\gamma_{k}$ are positive and that $q$ odd is the fixed cardinality of the finite field $\mathbb{F}_{q}$. Let $\mathfrak{P}=\left\{L\left(s, \chi_{P}\right): P \in \mathcal{P}_{2 g+1, q}\right\}$ to be the family of L-functions associated with the quadratic character $\chi_{P}$. Then with the same notation as before, we have

$$
\begin{aligned}
& \sum_{P \in \mathcal{P}_{2 g+1, q}} \frac{\prod_{k=1}^{K} L\left(\frac{1}{2}+\alpha_{k}, \chi_{P}\right)}{\prod_{m=1}^{Q} L\left(\frac{1}{2}+\gamma_{m}, \chi_{P}\right)} \\
& =\sum_{P \in \mathcal{P}_{2 g+1, q}} \sum_{\varepsilon \in\{-1,1\}^{k}}|P|^{-\frac{1}{2} \sum_{k=1}^{K}\left(\varepsilon_{k} \alpha_{k}-\alpha_{k}\right)} \prod_{k=1}^{K} \mathcal{X}\left(\frac{1}{2}+\frac{\alpha_{k}-\varepsilon_{k} \alpha_{k}}{2}\right) \\
& \quad \times Y_{\mathfrak{P}}\left(\varepsilon_{1} \alpha_{1}, \ldots, \varepsilon_{K} \alpha_{K} ; \gamma\right) A_{\mathfrak{P}}\left(\varepsilon_{1} \alpha_{1}, \ldots, \varepsilon_{K} \alpha_{K} ; \gamma\right)+o(P),
\end{aligned}
$$


where

$$
\begin{aligned}
A_{\mathfrak{P}}(\alpha ; \gamma)= & \prod_{\substack{P \text { monic } \\
\text { irreducible }}} \frac{\prod_{j \leq k \leq K}\left(1-\frac{1}{|P|^{1+\alpha_{j}+\alpha_{k}}}\right) \prod_{m \leq r \leq Q}\left(1-\frac{1}{|P|^{1+\gamma_{m}+\gamma_{r}}}\right)}{\prod_{k=1}^{K} \prod_{m=1}^{Q}\left(1-\frac{1}{|P|^{1+\alpha_{k}+\gamma_{m}}}\right)} \\
& \times\left(1+\sum_{0<\sum_{k} a_{k}+\sum_{m} c_{m} \text { is even }}^{|P|^{\sum_{k} a_{k}\left(\frac{1}{2}+\alpha_{k}\right)+\sum_{m} c_{m}\left(\frac{1}{2}+\gamma_{m}\right)}}\right)
\end{aligned}
$$

and

$$
Y_{\mathfrak{P}}(\alpha ; \gamma)=\frac{\prod_{j \leq k \leq K} \zeta_{A}\left(1+\alpha_{j}+\alpha_{k}\right) \prod_{m \leq r \leq Q} \zeta_{A}\left(1+\gamma_{m}+\gamma_{r}\right)}{\prod_{k=1}^{K} \prod_{m=1}^{Q} \zeta_{A}\left(1+\alpha_{k}+\gamma_{m}\right)}
$$

If we compare the above conjectures with the ones presented by Andrade and Keating in the previous section, one can immediately see that although they are similar in nature, there is an important difference between the formulas and the final shape of the conjectures. More specifically, one of the main differences is the arithmetic term that is produced in both conjectures. These factors are not the same and this is due to the fact that in one setting, we are averaging over square-free polynomials and so an Euler product is produced that needs to be carried out through the recipe and in the end produce the term $A_{k}$ in Andrade and Keating conjecture, while in the case presented in this paper, the average is taken over prime numbers and the final formula produces a simpler arithmetic factor due to precise formula that we have when using the prime polynomial theorem in $\mathbb{F}_{q}[T]$. This difference comes from the fourth step in the recipe when we replace each summand by its expected value.

In the following sections, we present the details of how to arrive at these conjectures.

\section{Integral moments of $L$-functions over prime polynomials}

In this section, we present the details of the recipe for conjecturing moments of the family of quadratic Dirichlet $L$-function $L\left(s, \chi_{P}\right)$ associated to hyperelliptic curves of genus $g$ over fixed finite field $\mathbb{F}_{q}$ as $g \rightarrow \infty$. As in Andrade and Keating [4], we will adjust the recipe first presented in [7] to the function field setting.

Let $P \in \mathcal{P}_{2 g+1, q}$. For a fixed $k$, we aim to obtain an asymptotic expression for

$$
\sum_{P \in \mathcal{P}_{2 g+1, q}} L\left(\frac{1}{2}, \chi_{P}\right)^{k}
$$


as $g \rightarrow \infty$. In order to achieve this, we consider the more general expression obtained by introducing small shifts, say $\alpha_{1}, \ldots, \alpha_{k}$

$$
\sum_{P \in \mathcal{P}_{2 g}+1, q} L\left(\frac{1}{2}+\alpha_{1}, \chi_{P}\right) \cdots L\left(\frac{1}{2}+\alpha_{k}, \chi_{P}\right) .
$$

Introducing the shifts helps to reveal the hidden structures in the form of symmetries. Moreover, the calculations are simplified by the removal of higher-order poles. In the end, letting each $\alpha_{1}, \cdots, \alpha_{k}$ tend to 0 will provide an asymptotic formula for (3.1).

\subsection{Analogies between classical $L$-functions and $L$-functions over function fields}

The first step to obtaining a conjecture for the integral moments of $L$-functions of any family is the use of the approximate functional equation. Thus, the "approximate" functional equation for the $L$-function attached to the character $\chi_{P}$ is given by

$$
L\left(s, \chi_{P}\right)=\sum_{\substack{n \text { monic } \\ \operatorname{deg}(n) \leq g}} \frac{\chi_{P}(n)}{|n|^{s}}+\mathcal{X}_{p}(s) \sum_{\substack{n \text { monic } \\ \operatorname{deg}(n) \leq g-1}} \frac{\chi_{P}(n)}{|n|^{1-s}},
$$

where $P \in \mathcal{P}_{2 g+1, q}$ and $\mathcal{X}_{P}(s)=q^{g(1-2 s)}$. Note that $\mathcal{X}_{P}(s)$ can also be re-written as follows:

$$
\mathcal{X}_{P}(s)=|P|^{\frac{1}{2}-s} \mathcal{X}(s),
$$

where $\mathcal{X}(s)=q^{-\frac{1}{2}+s}$ corresponds to the gamma factor that appears in the classical quadratic $L$-functions.

The next result, quoted from [4], makes the analogy between the function field case and the number field case more apparent.

Lemma 3.1 We have that

$$
\mathcal{X}_{P}(s)^{\frac{1}{2}}=\mathcal{X}_{P}(1-s)^{-\frac{1}{2}}
$$

and

$$
\mathcal{X}_{P}(s) \mathcal{X}_{P}(1-s)=1
$$

Consider the following completed $L$-function:

$$
Z_{\mathcal{L}}\left(s, \chi_{P}\right)=\mathcal{X}_{P}(s)^{-\frac{1}{2}} L\left(s, \chi_{P}\right)
$$

We will apply the recipe to this completed $L$-function, since it simplifies the calculations and satisfies a more symmetric functional equation given by the next lemma.

Lemma 3.2 Let $Z_{\mathcal{L}}\left(s, \chi_{P}\right)$ be the $Z$-function defined above, then we have the following functional equation:

$$
Z_{\mathcal{L}}\left(s, \chi_{P}\right)=Z_{\mathcal{L}}\left(1-s, \chi_{P}\right)
$$

Proof Direct from the definition of $Z_{\mathcal{L}}\left(s, \chi_{P}\right)$ and Lemma 3.1. 
Now, let

$$
L_{P}(s)=\sum_{P \in \mathcal{P}_{2 g+1, q}} Z\left(s ; \alpha_{1}, \ldots, \alpha_{k}\right)
$$

be the $k$-shifted moment, with

$$
Z\left(s ; \alpha_{1}, \ldots, \alpha_{k}\right)=\prod_{i=1}^{k} Z_{\mathcal{L}}\left(s+\alpha_{i}, \chi_{P}\right)
$$

Using the "approximate" functional equation (3.3) and Lemma 3.1, we have

$$
Z_{\mathcal{L}}\left(s, \chi_{P}\right)=\mathcal{X}_{P}(s)^{-\frac{1}{2}} \sum_{\substack{n \text { monic } \\ \operatorname{deg}(n) \leq g}} \frac{\chi_{P}(n)}{|n|^{s}}+\mathcal{X}_{p}(1-s)^{-\frac{1}{2}} \sum_{\substack{n \text { monic } \\ \operatorname{deg}(n) \leq g-1}} \frac{\chi_{P}(n)}{|n|^{1-s}}
$$

\subsection{Adapting the CFKRS recipe for the function field case}

We present the steps of the recipe which follows from [4,7] with the necessary modifications for the family of $L\left(s, \chi_{P}\right)$.

(1) Write the product of $k$-shifted $L$-functions.

$$
Z\left(\frac{1}{2} ; \alpha_{1}, \ldots, \alpha_{k}\right)=Z_{\mathcal{L}}\left(\frac{1}{2}+\alpha_{1}, \chi_{P}\right) \cdots Z_{\mathcal{L}}\left(\frac{1}{2}+\alpha_{k}, \chi_{P}\right)
$$

(2) Replace each $L$-function with the two terms from its approximate functional equation (3.3) with $s=1 / 2+\alpha_{i}$.

$$
Z\left(\frac{1}{2} ; \alpha_{1}, \ldots, \alpha_{k}\right)=\sum_{\varepsilon_{i}= \pm 1} \prod_{i=1}^{k} \mathcal{X}_{P}\left(\frac{1}{2}+\varepsilon_{i} \alpha_{i}\right)^{-\frac{1}{2}} \sum_{\substack{n_{1}, \ldots, n_{k} \\ \operatorname{deg}\left(n_{i}\right) \leq f\left(\epsilon_{i}\right)}} \frac{\chi_{P}\left(n_{1} \cdots n_{k}\right)}{\prod_{i=1}^{k}\left|n_{i}\right|^{\frac{1}{2}+\varepsilon_{i} \alpha_{i}}}
$$

where $f(1)=g$, and $f(-1)=g-1$.

(3) Replace each product of $\varepsilon_{f}$-factors by its expected value when averaged over $\mathcal{P}_{2 g+1, q}$.

In our case, $\varepsilon_{f}$-factors are equal to 1 . Thus, the product will not appear and will not affect the result.

(4) Replace each summand by its expected value when averaged over $\mathcal{P}_{2 g+1, q}$.

We need first to average over all primes $P \in \mathcal{P}_{2 g+1, q}$. The next lemma gives the orthogonality relation for these quadratic Dirichlet characters over function fields.

\section{Lemma 3.3}

$$
\lim _{\operatorname{deg}(P) \rightarrow \infty} \frac{1}{\# \mathcal{P}_{2 g+1, q}} \sum_{P \in \mathcal{P}_{2 g+1, q}} \chi_{P}(n)= \begin{cases}1 & \text { if } n=\square \\ 0 & \text { otherwise }\end{cases}
$$


Proof Consider the case when $n=\square$, then we have

$$
\sum_{P \in \mathcal{P}_{2 g+1, q}} \chi_{P}(n)=\sum_{P \in \mathcal{P}_{2 g+1, q}} \chi_{P}\left(l^{2}\right)=\sum_{\substack{P \in \mathcal{P}_{2 g+1, q} \\ P \nmid l}} 1,
$$

since we are summing over primes of degree $2 g+1$ and $P \nmid l$, and $\operatorname{deg}(l) \leq 2 g$, which means that we are counting all primes of degree $2 g+1$, thus,

$$
\sum_{\substack{P \in \mathcal{P}_{2 g+1, q} \\ P \nmid l}} 1=\# \mathcal{P}_{2 g+1, q}
$$

Hence, if $n$ is a square of a polynomial,

$$
\lim _{\operatorname{deg}(P) \rightarrow \infty} \frac{1}{\# \mathcal{P}_{2 g+1, q}} \sum_{P \in \mathcal{P}_{2 g+1, q}} \chi_{P}(n)=1
$$

It remains to consider the case when $n \neq \square$, Rudnick [30] shows that

$$
\left|\sum_{P \in \mathcal{P}_{2 g+1, q}} \chi_{P}(n)\right| \ll \frac{|P|^{\frac{1}{2}}}{\log _{q}|P|} \operatorname{deg}(n),
$$

and from Polynomial Prime Theorem (2.1), we have

$$
\frac{1}{\# \mathcal{P}_{2 g+1, q}} \sum_{P \in \mathcal{P}_{2 g+1, q}} \chi_{P}(n) \ll|P|^{-\frac{1}{2}} \operatorname{deg}(n) .
$$

Hence, if $n$ is not a square of a polynomial, we have that

$$
\lim _{\operatorname{deg}(P) \rightarrow \infty} \frac{1}{\# \mathcal{P}_{2 g+1, q}} \sum_{P \in \mathcal{P}_{2 g+1, q}} \chi_{P}(n)=0 .
$$

Using Lemma 3.3, we can average the summand in (3.13) that is

$$
\begin{aligned}
& \lim _{\operatorname{deg}(P) \rightarrow \infty} \frac{1}{\# \mathcal{P}_{2 g+1, q}} \sum_{P \in \mathcal{P}_{2 g+1, q}} \sum_{n_{1}, \ldots, n_{k}} \frac{\chi_{P}\left(n_{1} \cdots n_{k}\right)}{\prod_{i=1}^{k}\left|n_{i}\right|^{\frac{1}{2}+\varepsilon_{i} \alpha_{i}}} \\
& =\sum_{m \text { monic }} \sum_{\substack{n_{1}, \ldots, n_{k} \\
n_{1} \cdots n_{k}=m^{2}}} \frac{1}{\prod_{i=1}^{k}\left|n_{i}\right|^{\frac{1}{2}+\varepsilon_{i} \alpha_{i}}}
\end{aligned}
$$


(5) Let each $n_{1}, \ldots, n_{k}$ to be monic polynomials and call the total result $M_{f}\left(s, \alpha_{1}, \ldots, \alpha_{k}\right)$ to produce the desired conjecture.

If we let

$$
R_{k}\left(\frac{1}{2} ; \varepsilon_{1} \alpha_{1}, \ldots, \varepsilon_{k} \alpha_{k}\right)=\sum_{m \text { monic }} \sum_{\substack{n_{1}, \ldots, n_{k} \\ n_{i} \text { monic } \\ n_{1} \cdots n_{k}=m^{2}}} \frac{1}{\prod_{i=1}^{k}\left|n_{i}\right|^{\frac{1}{2}+\varepsilon_{i} \alpha_{i}}}
$$

then the extended sum produced by the recipe is

$$
M\left(\frac{1}{2} ; \alpha_{1}, \ldots, \alpha_{k}\right)=\sum_{\varepsilon_{i}= \pm 1} \prod_{i=1}^{k} \chi_{P}\left(\frac{1}{2}+\varepsilon_{i} \alpha_{i}\right)^{-\frac{1}{2}} R_{k}\left(\frac{1}{2} ; \varepsilon_{1} \alpha_{1}, \ldots, \varepsilon_{k} \alpha_{k}\right)
$$

(6) The conclusion is

$$
\sum_{P \in \mathcal{P}_{2 g+1, q}} Z\left(\frac{1}{2} ; \alpha_{1}, \ldots, \alpha_{k}\right)=\sum_{P \in \mathcal{P}_{2 g+1, q}} M\left(\frac{1}{2}, \alpha_{1}, \ldots, \alpha_{k}\right)(1+o(1)) .
$$

\subsection{Putting the conjecture in a more useful form}

In this section, we put the conjecture (3.24) in a more useful form, we write $R_{k}$ as an Euler product, then factor out the appropriate $\zeta_{A}(s)$-factors. Let

$$
\psi(x):=\sum_{\substack{n_{1}, \ldots, n_{k} \\ n_{i} \text { monic } \\ n_{1} \cdots n_{k}=x}} \frac{1}{\left|n_{1}\right|^{s+\alpha_{1}} \cdots\left|n_{k}\right|^{s+\alpha_{k}}}
$$

then it is easy to see that $\psi\left(m^{2}\right)$ is multiplicative on $m$. We can write $R_{k}\left(s ; \alpha_{1}, \ldots, \alpha_{k}\right)$ as follows:

$$
\begin{aligned}
R_{k}\left(s ; \alpha_{1}, \ldots, \alpha_{k}\right) & =\sum_{m \text { monic }} \psi\left(m^{2}\right) \\
& =\prod_{\substack{P \text { monic } \\
\text { irreducible }}}\left(1+\sum_{j=1}^{\infty} \psi\left(P^{2 j}\right)\right),
\end{aligned}
$$

where

$$
\psi\left(P^{2 j}\right)=\sum_{\substack{n_{1}, \ldots, n_{k} \\ n_{i} \text { monic } \\ n_{1} \cdots n_{k}=P^{2 j}}} \frac{1}{\left|n_{1}\right|^{s+\alpha_{1}} \cdots\left|n_{k}\right|^{s+\alpha_{k}}}
$$


Since we have $n_{1} \cdots n_{k}=P^{2 j}$, then for each $i=1, \cdots, k$, write $n_{i}$ as $n_{i}=P^{e_{i}}$, for some $e_{i} \geq 0$ and $e_{1}+\cdots+e_{k}=2 j$, and (3.27) becomes

$$
\psi\left(P^{2 j}\right)=\sum_{\substack{e_{1}, \ldots, e_{k} \geq 0 \\ e_{1}+\cdots+e_{k}=2 j}} \prod_{i=1}^{k} \frac{1}{|P|^{e_{i}\left(s+\alpha_{i}\right)}}
$$

and so, we have

$$
R_{k}\left(s ; \alpha_{1}, \ldots, \alpha_{k}\right)=\prod_{\substack{P \text { monic } \\ \text { irreducible }}}\left(1+\sum_{j=1}^{\infty} \sum_{\substack{e_{1}, \ldots, e_{k} \geq 0 \\ e_{1}+\cdots+e_{k}=2 j}} \prod_{i=1}^{k} \frac{1}{|P|^{e_{i}\left(s+\alpha_{i}\right)}}\right)
$$

One can see that when $\alpha_{i}=0$ and $s=1 / 2$, the poles only arise from the terms with $e_{1}+\cdots+e_{k}=2$. Define $R_{k, P}\left(s ; \alpha_{1}, \cdots, \alpha_{k}\right)$ to be as follows:

$$
\begin{aligned}
R_{k, P}\left(s ; \alpha_{1}, \ldots, \alpha_{k}\right) & =1+\sum_{j=1}^{\infty} \sum_{\substack{e_{1}, \ldots, e_{k} \geq 0 \\
e_{1}+\cdots+e_{k}=2 j}} \prod_{i=1}^{k} \frac{1}{|P|^{e_{i}\left(s+\alpha_{i}\right)}} \\
& =1+\sum_{\substack{e_{1}, \ldots, e_{k} \geq 0 \\
e_{1}+\cdots+e_{k}=2}} \prod_{i=1}^{k} \frac{1}{|P|^{e_{i}\left(s+\alpha_{i}\right)}}+\text { (lower-order terms) } \\
& =1+\sum_{1 \leq i \leq j \leq k} \frac{1}{|P|^{2 s+\alpha_{i}+\alpha_{j}}}+O\left(|P|^{-4 s+\epsilon}\right)
\end{aligned}
$$

for $\mathfrak{R}\left(\alpha_{i}\right)$ small enough (see [7] for more details). And so, we have

$$
\begin{aligned}
R_{k, P} & \left(s ; \alpha_{1}, \ldots, \alpha_{k}\right) \\
& =\prod_{1 \leq i \leq j \leq k}\left(1+\frac{1}{|P|^{2 s+\alpha_{i}+\alpha_{j}}}\right) \times\left(1+O\left(|P|^{-4 s+\epsilon}\right)\right) .
\end{aligned}
$$

Recall that

$$
\frac{\zeta_{A}(2 s)}{\zeta_{A}(4 s)}=\prod_{\substack{P \text { monic } \\ \text { irreducible }}}\left(1+\frac{1}{|P|^{2 s}}\right)
$$

has a simple pole as $s=1 / 2$. Therefore,

$$
\prod_{\substack{P \text { monic } \\ \text { irreducible }}}\left(1+O\left(|P|^{-4 s+\epsilon}\right)\right)
$$

is analytic in $\Re(s)>1 / 4$, and $\prod_{P} R_{k, P}$ has a pole at $s=1 / 2$ of order $k(k+1) / 2$ if $\alpha_{i}=0$ for all $i=1, \ldots, k$. It remains to factor out the appropriate zeta-factors. Since 
we have

$$
R_{k}\left(s ; \alpha_{1}, \ldots, \alpha_{k}\right)=\prod_{\substack{P \text { monic } \\ \text { irreducible }}} R_{k, P}\left(s ; \alpha_{1}, \ldots, \alpha_{k}\right)
$$

then from (3.31) and (3.32), we can write

$$
R_{k}\left(s ; \alpha_{1}, \ldots, \alpha_{k}\right)=\prod_{1 \leq i \leq j \leq k} \zeta_{A}\left(2 s+\alpha_{i}+\alpha_{j}\right) A_{k}\left(s ; \alpha_{1}, \ldots, \alpha_{k}\right),
$$

where

$$
\begin{aligned}
A_{k}\left(s ; \alpha_{1}, \ldots, \alpha_{k}\right) & \\
& =\prod_{\substack{P \text { monic } \\
\text { irreducible }}}\left(R_{k, P}\left(s ; \alpha_{1}, \ldots, \alpha_{k}\right) \prod_{1 \leq i \leq j \leq k}\left(1-\frac{1}{|P|^{2 s+\alpha_{i}+\alpha_{j}}}\right)\right) .
\end{aligned}
$$

Notice that for some $\delta>0$ and for all $\alpha_{i}$ 's in some sufficiently small neighbourhood of $0, A_{k}$ is an absolutely convergent Dirichlet series for $\mathfrak{R}(s)>1 / 2+\delta$. Combining (3.23) and (3.35), we have

$$
\begin{aligned}
M\left(\frac{1}{2} ; \alpha_{1}, \ldots, \alpha_{k}\right)= & \sum_{\varepsilon_{i}= \pm 1} \prod_{i=1}^{k} \mathcal{X}_{P}\left(\frac{1}{2}+\varepsilon_{i} \alpha_{i}\right)^{-\frac{1}{2}} \prod_{1 \leq i \leq j \leq k} \zeta_{A}\left(1+\alpha_{i}+\alpha_{j}\right) \\
& \times A_{k}\left(\frac{1}{2} ; \varepsilon_{1} \alpha_{1}, \ldots, \varepsilon_{k} \alpha_{k}\right)
\end{aligned}
$$

Hence,

$$
\begin{aligned}
\sum_{P \in \mathcal{P}_{2 g+1, q}} Z\left(\frac{1}{2} ; \alpha_{1}, \ldots, \alpha_{k}\right) \\
=\sum_{P \in \mathcal{P}_{2 g+1, q}} \sum_{\varepsilon_{i}= \pm 1} \prod_{i=1}^{k} \mathcal{X}_{P}\left(\frac{1}{2}+\varepsilon_{i} \alpha_{i}\right)^{-\frac{1}{2}} A_{k}\left(\frac{1}{2} ; \varepsilon_{1} \alpha_{1}, \ldots, \varepsilon_{k} \alpha_{k}\right) \\
\quad \times \prod_{1 \leq i \leq j \leq k} \zeta_{A}\left(1+\alpha_{i}+\alpha_{j}\right)(1+o(1)) .
\end{aligned}
$$

From the definition of $\mathcal{X}_{P}(s)$ in (3.4), we have

$$
\mathcal{X}_{P}\left(\frac{1}{2}+\varepsilon_{i} \alpha_{i}\right)^{-\frac{1}{2}}=|P|^{\frac{\varepsilon_{i} \alpha_{i}}{2}} \mathcal{X}\left(\frac{1}{2}+\varepsilon_{i} \alpha_{i}\right)^{-\frac{1}{2}}
$$


Hence,

$$
\begin{aligned}
\sum_{P \in \mathcal{P}_{2 g+1, q}} Z\left(\frac{1}{2} ; \alpha_{1}, \cdots, \alpha_{k}\right) & \\
= & \sum_{\varepsilon_{i}= \pm 1} \prod_{i=1}^{k} \mathcal{X}\left(\frac{1}{2}+\varepsilon_{i} \alpha_{i}\right)^{-\frac{1}{2}} \sum_{P \in \mathcal{P}_{2 g+1, q}} R_{k}\left(\frac{1}{2} ; \varepsilon_{1} \alpha_{1}, \cdots, \varepsilon_{k} \alpha_{k}\right) \\
& \times|P|^{\frac{1}{2} \sum_{i=1}^{k} \varepsilon_{i} \alpha_{i}}(1+o(1))
\end{aligned}
$$

We finish this section writing $A_{k}$ as an Euler product in the following lemma.

Lemma 3.4 We have

$$
\begin{aligned}
A_{k}\left(\frac{1}{2} ; \alpha_{1}, \ldots, \alpha_{k}\right)= & \prod_{\substack{P \text { monic } \\
\text { irreducible }}} \prod_{1 \leq i \leq j \leq k}\left(1-\frac{1}{|P|^{1+\alpha_{i}+\alpha_{j}}}\right) \\
& \times \frac{1}{2}\left(\prod_{i=1}^{k}\left(1-\frac{1}{|P|^{1 / 2+\alpha_{i}}}\right)^{-1}+\prod_{i=1}^{k}\left(1+\frac{1}{|P|^{1 / 2+\alpha_{i}}}\right)^{-1}\right) .
\end{aligned}
$$

Proof Applying $A_{k}\left(s ; \alpha_{1}, \ldots, \alpha_{k}\right)$ and $R_{k, P}\left(s ; \alpha_{1}, \ldots, \alpha_{k}\right)$ in (3.36) and (3.30) for $s=1 / 2$, we have

$$
\begin{aligned}
& A_{k}\left(\frac{1}{2} ; \alpha_{1}, \ldots, \alpha_{k}\right) \\
& \quad=\prod_{\substack{P \text { monic } \\
\text { irreducible }}} \prod_{1 \leq i \leq j \leq k}\left(1-\frac{1}{|P|^{1+\alpha_{i}+\alpha_{j}}}\right)\left(1+\sum_{j=1}^{\infty} \sum_{\substack{e_{1}, \ldots, e_{k} \geq 0 \\
e_{1}+\cdots+e_{k}=2 j}} \prod_{i=1}^{k} \frac{1}{|P|^{e_{i}\left(1 / 2+\alpha_{i}\right)}}\right) .
\end{aligned}
$$

By simplifying the second brackets, we obtain the result in the lemma, i.e.

$$
\begin{aligned}
1 & +\sum_{j=1}^{\infty} \sum_{\substack{e_{1}, \cdots, e_{k} \geq 0 \\
e_{1}+\cdots+e_{k}=2 j}} \prod_{i=1}^{k}\left(\frac{1}{|P|^{\left(1 / 2+\alpha_{i}\right)}}\right)^{e_{i}} \\
& =\sum_{j=0}^{\infty} \frac{1}{2}\left(2 \sum_{\substack{e_{1}, \cdots, e_{k} \geq 0 \\
e_{1}+\cdots+e_{k}=2 j}} \prod_{i=1}^{k}\left(\frac{1}{|P|^{\left(1 / 2+\alpha_{i}\right)}}\right)^{e_{i}}\right) \\
& =\frac{1}{2}\left(\prod_{i=1}^{k} \sum_{e_{i}=0}^{\infty}\left(\frac{1}{|P|^{\left(1 / 2+\alpha_{i}\right)}}\right)^{e_{i}}+\prod_{i=1}^{k} \sum_{e_{i}=0}^{\infty}(-1)^{e_{i}}\left(\frac{1}{|P|^{\left(1 / 2+\alpha_{i}\right)}}\right)^{e_{i}}\right) \\
& =\frac{1}{2}\left(\prod_{i=1}^{k}\left(1-\frac{1}{|P|^{1 / 2+\alpha_{i}}}\right)^{-1}+\prod_{i=1}^{k}\left(1+\frac{1}{|P|^{1 / 2+\alpha_{i}}}\right)^{-1}\right) .
\end{aligned}
$$




\subsection{The contour integral representation of the conjecture}

We begin this section with Lemma 2.5.2 from [7], which helps write our conjecture as a contour integral.

Lemma 3.5 Suppose $F$ is a symmetric function of $k$ variables, regular near $(0, \ldots, 0)$, and that $f(s)$ has a simple pole $s=0$ of residue 1 and is otherwise analytic in a neighbourhood of $s=0$, and let

$$
K\left(a_{1}, \ldots, a_{k}\right)=F\left(a_{1}, \ldots, a_{k}\right) \prod_{1 \leq i \leq j \leq k} f\left(a_{i}+a_{j}\right),
$$

or

$$
K\left(a_{1}, \ldots, a_{k}\right)=F\left(a_{1}, \ldots, a_{k}\right) \prod_{1 \leq i<j \leq k} f\left(a_{i}+a_{j}\right)
$$

If $\alpha_{i}+\alpha_{j}$ are contained in the region of analyticity of $f(s)$, then

$$
\begin{aligned}
\sum_{\varepsilon_{i}= \pm 1} K\left(\varepsilon_{1} a_{1}, \ldots, \varepsilon_{k} a_{k}\right)= & \frac{(-1)^{k(k-1) / 2}}{(2 \pi i)^{k}} \frac{2^{k}}{k !} \oint \cdots \oint K\left(z_{1}, \ldots, z_{k}\right) \\
& \times \frac{\Delta\left(z_{1}^{2}, \ldots, z_{k}^{2}\right)^{2} \prod_{i=1}^{k} z_{i}}{\prod_{i=1}^{k} \prod_{j=1}^{k}\left(z_{i}-\alpha_{j}\right)\left(z_{i}+\alpha_{j}\right)} \mathrm{d} z_{1} \cdots \mathrm{d} z_{k}
\end{aligned}
$$

and

$$
\begin{aligned}
& \sum_{\varepsilon_{i}= \pm 1}\left(\prod_{i=1}^{k} \varepsilon_{i}\right) K\left(\varepsilon_{1} a_{1}, \ldots, \varepsilon_{k} a_{k}\right) \\
& =\frac{(-1)^{k(k-1) / 2}}{(2 \pi i)^{k}} \frac{2^{k}}{k !} \oint \cdots \oint K\left(z_{1}, \ldots, z_{k}\right) \\
& \quad \times \frac{\Delta\left(z_{1}^{2}, \cdots, z_{k}^{2}\right)^{2} \prod_{i=1}^{k} \alpha_{i}}{\prod_{i=1}^{k} \prod_{j=1}^{k}\left(z_{i}-\alpha_{j}\right)\left(z_{i}+\alpha_{j}\right)} \mathrm{d} z_{1} \cdots \mathrm{d} z_{k},
\end{aligned}
$$

where the path of the integration encloses the $\pm \alpha_{i}$ s.

Recall that

$$
\begin{aligned}
& \sum_{P \in \mathcal{P}_{2 g+1, q}} Z\left(\frac{1}{2} ; \alpha_{1}, \ldots, \alpha_{k}\right) \\
& =\sum_{P \in \mathcal{P}_{2 g+1, q}} \prod_{i=1}^{k} \mathcal{X}_{P}\left(\frac{1}{2}+\alpha_{i}\right)^{-\frac{1}{2}} L\left(\frac{1}{2}+\alpha_{i}, \chi_{P}\right),
\end{aligned}
$$


where $\mathcal{X}_{P}(s)$ is defined in (3.4). Since $\mathcal{X}_{P}\left(\frac{1}{2}+\alpha_{i}\right)^{-\frac{1}{2}}$ does not depend on $P$, we can factor out it, and from (3.48) and (3.40), we have

$$
\begin{aligned}
& \sum_{P \in \mathcal{P}_{2 g+1, q}} \prod_{i=1}^{k} L\left(\frac{1}{2}+\alpha_{i}, \chi_{P}\right) \\
& =\sum_{P \in \mathcal{P}_{2 g+1, q}}|P|^{-\frac{1}{2} \sum_{i=0}^{k} \alpha_{i}} \prod_{i=1}^{k} \mathcal{X}\left(\frac{1}{2}+\alpha_{i}\right)^{\frac{1}{2}} \sum_{\varepsilon_{i}= \pm 1} \prod_{i=1}^{k} \mathcal{X}\left(\frac{1}{2}+\varepsilon_{i} \alpha_{i}\right)^{-\frac{1}{2}} \\
& \quad \times A_{k}\left(\frac{1}{2} ; \alpha_{1}, \cdots, \alpha_{k}\right)|P|^{\frac{1}{2} \sum_{i=0}^{k} \varepsilon_{i} \alpha_{i}} \\
& \quad \times \prod_{1 \leq i<j \leq k} \zeta_{A}\left(1+\varepsilon_{i} \alpha_{i}+\varepsilon_{j} \alpha_{j}\right)(1+o(1))
\end{aligned}
$$

From each term in the second product, we factor out $(\log q)^{-1}$ to get

$$
\begin{aligned}
& \sum_{P \in \mathcal{P}_{2 g+1, q}} \prod_{i=1}^{k} L\left(\frac{1}{2}+\alpha_{i}, \chi_{P}\right) \\
& =\sum_{P \in \mathcal{P}_{2 g+1, q}} \frac{|P|^{-\frac{1}{2} \sum_{i=0}^{k} \alpha_{i}} \prod_{i=1}^{k} \mathcal{X}\left(\frac{1}{2}+\alpha_{i}\right)^{\frac{1}{2}}}{(\log q)^{k(k+1) / 2}} \sum_{\varepsilon_{i}= \pm 1} \prod_{i=1}^{k} \mathcal{X}\left(\frac{1}{2}+\varepsilon_{i} \alpha_{i}\right)^{-\frac{1}{2}} \\
& \quad \times A_{k}\left(\frac{1}{2} ; \alpha_{1}, \ldots, \alpha_{k}\right)|P|^{\frac{1}{2} \sum_{i=0}^{k} \varepsilon_{i} \alpha_{i}} \\
& \quad \times \prod_{1 \leq i<j \leq k} \zeta_{A}\left(1+\varepsilon_{i} \alpha_{i}+\varepsilon_{j} \alpha_{j}\right)(\log q)(1+o(1))
\end{aligned}
$$

Now, call

$$
F\left(\alpha_{1}, \ldots, \alpha_{k}\right)=\prod_{i=1}^{k} \mathcal{X}\left(\frac{1}{2}+\alpha_{i}\right)^{-\frac{1}{2}} A_{k}\left(\frac{1}{2} ; \alpha_{i}, \ldots, \alpha_{k}\right)|P|^{\frac{1}{2} \sum_{i=1}^{k} \alpha_{i}}
$$

and

$$
f(s)=\zeta_{A}(1+s) \log q \text { and so } f\left(\alpha_{i}+\alpha_{j}\right)=\zeta_{A}\left(1+\alpha_{i}+\alpha_{j}\right) \log q,
$$

where $f(s)$ has a simple pole at $s=0$ with residue 1 .

If we denote

$$
K\left(\alpha_{1}, \ldots, \alpha_{k}\right)=F\left(\alpha_{1}, \ldots, \alpha_{k}\right) \prod_{1 \leq i \leq j \leq k} f\left(\alpha_{i}+\alpha_{j}\right)
$$


then (3.50) can be written as follows:

$$
\begin{aligned}
& \sum_{P \in \mathcal{P}_{2 g+1, q}} \prod_{i=1}^{k} L\left(\frac{1}{2}+\alpha_{i}, \chi_{P}\right) \\
& =\sum_{P \in \mathcal{P}_{2 g+1, q}} \frac{\prod_{i=1}^{k}|P|^{-\frac{1}{2} \sum_{i=0}^{k} \alpha_{i}} \mathcal{X}\left(\frac{1}{2}+\alpha_{i}\right)^{\frac{1}{2}}}{(\log q)^{k(k+1) / 2}} \\
& \quad \times \sum_{\varepsilon_{i}= \pm 1} K\left(\varepsilon_{1} \alpha_{1}, \ldots, \varepsilon_{k} \alpha_{k}\right)(1+o(1)) .
\end{aligned}
$$

Using Lemma 3.5, we have

$$
\begin{aligned}
& \sum_{P \in \mathcal{P}_{2 g+1, q}} \prod_{i=1}^{k} L\left(\frac{1}{2}+\alpha_{i}, \chi_{P}\right) \\
& =\sum_{P \in \mathcal{P}_{2 g+1, q}} \frac{\prod_{i=1}^{k}|P|^{-\frac{1}{2} \sum_{i=0}^{k} \alpha_{i}} \mathcal{X}\left(\frac{1}{2}+\alpha_{i}\right)^{\frac{1}{2}}}{(\log q)^{k(k+1) / 2}} \frac{(-1)^{k(k-1) / 2}}{(2 \pi i)^{k}} \frac{2^{k}}{k !} \\
& \times \oint \cdots \oint K\left(z_{1}, \ldots, z_{k}\right) \frac{\Delta\left(z_{1}^{2}, \ldots, z_{k}^{2}\right)^{2} \prod_{i=1}^{k} z_{i}}{\prod_{i=1}^{k} \prod_{j=1}^{k}\left(z_{i}-\alpha_{j}\right)\left(z_{i}+\alpha_{j}\right)} \mathrm{d} z_{1} \cdots \mathrm{d} z_{k} \\
& \times(1+o(1)) \\
& =\sum_{P \in \mathcal{P}_{2 g+1, q}} \prod_{i=1}^{k}|P|^{-\frac{1}{2} \sum_{i=0}^{k} \alpha_{i}} \mathcal{X}\left(\frac{1}{2}+\alpha_{i}\right)^{\frac{1}{2}} \frac{(-1)^{k(k-1) / 2}}{(2 \pi i)^{k}} \frac{2^{k}}{k !} \\
& \times \oint \cdots \oint F\left(z_{1}, \ldots, z_{k}\right) \prod_{1 \leq i \leq j \leq k} \zeta_{A}\left(1+\varepsilon_{i} \alpha_{i}+\varepsilon_{j} \alpha_{j}\right) \\
& \times \frac{\Delta\left(z_{1}^{2}, \ldots, z_{k}^{2}\right)^{2} \prod_{i=1}^{k} z_{i}}{\prod_{i=1}^{k} \prod_{j=1}^{k}\left(z_{i}-\alpha_{j}\right)\left(z_{i}+\alpha_{j}\right)} \mathrm{d} z_{1} \cdots \mathrm{d} z_{k}+o(|P|) \\
& =\sum_{P \in \mathcal{P}_{2 g+1, q}} \prod_{i=1}^{k}|P|^{-\frac{1}{2} \sum_{i=0}^{k} \alpha_{i}} \mathcal{X}\left(\frac{1}{2}+\alpha_{i}\right)^{\frac{1}{2}} \frac{(-1)^{k(k-1) / 2}}{(2 \pi i)^{k}} \frac{2^{k}}{k !} \\
& \times \oint \cdots \oint K\left(z_{1}, \ldots, z_{k}\right) \frac{\Delta\left(z_{1}^{2}, \ldots, z_{k}^{2}\right)^{2} \prod_{i=1}^{k} z_{i}}{\prod_{i=1}^{k} \prod_{j=1}^{k}\left(z_{i}-\alpha_{j}\right)\left(z_{i}+\alpha_{j}\right)} \mathrm{d} z_{1} \cdots d z_{k} \\
& +o(|P|)
\end{aligned}
$$

with

$$
K\left(z_{1}, \ldots, z_{k}\right)=F\left(z_{1}, \ldots, z_{k}\right) \prod_{1 \leq i \leq j \leq k} \zeta_{A}\left(1+\varepsilon_{i} \alpha_{i}+\varepsilon_{j} \alpha_{j}\right)
$$


Moreover, if we denote

$$
G\left(z_{1}, \ldots, z_{k}\right)=\prod_{i=1}^{k} \mathcal{X}\left(\frac{1}{2}+\alpha_{i}\right)^{-\frac{1}{2}} A_{k}\left(\frac{1}{2} ; \alpha_{i}, \ldots, \alpha_{k}\right) \prod_{1 \leq i \leq j \leq k} \zeta_{A}\left(1+z_{i}+z_{j}\right)
$$

then (3.55) becomes

$$
\begin{aligned}
& \sum_{P \in \mathcal{P}_{2 g+1, q}} \prod_{i=1}^{k}|P|^{-\frac{1}{2} \sum_{i=0}^{k} \alpha_{i}} \mathcal{X}\left(\frac{1}{2}+\alpha_{i}\right)^{\frac{1}{2}} \frac{(-1)^{k(k-1) / 2}}{(2 \pi i)^{k}} \frac{2^{k}}{k !} \\
& \times \oint \ldots \oint G\left(z_{1}, \ldots, z_{k}\right)|P|^{\frac{1}{2} \sum_{i=0}^{k} z_{i}} \frac{\Delta\left(z_{1}^{2}, \ldots, z_{k}^{2}\right)^{2} \prod_{i=1}^{k} z_{i}}{\prod_{i=1}^{k} \prod_{j=1}^{k}\left(z_{i}-\alpha_{j}\right)\left(z_{i}+\alpha_{j}\right)} \mathrm{d} z_{1} \cdots \mathrm{d} z_{k} \\
& +o(|P|) .
\end{aligned}
$$

Now, letting $\alpha_{i} \rightarrow 0$, we have

$$
\begin{aligned}
& \sum_{P \in \mathcal{P}_{2 g+1, q}} L\left(\frac{1}{2}, \chi_{P}\right)^{k} \\
& =\sum_{P \in \mathcal{P}_{2 g+1, q}} \frac{(-1)^{k(k-1) / 2}}{(2 \pi i)^{k}} \frac{2^{k}}{k !} \oint \cdots \oint G\left(z_{1}, \ldots, z_{k}\right)|P|^{\frac{1}{2} \sum_{i=0}^{k} z_{i}} \\
& \quad \times \frac{\Delta\left(z_{1}^{2}, \ldots, z_{k}^{2}\right)^{2} \prod_{i=1}^{k} z_{i}}{\prod_{i=1}^{k} z_{i}^{2 k}} \mathrm{~d} z_{1} \cdots \mathrm{d} z_{k}+o(|P|) .
\end{aligned}
$$

Calling

$$
\begin{aligned}
Q_{k}(x)= & \frac{(-1)^{k(k-1) / 2}}{(2 \pi i)^{k}} \frac{2^{k}}{k !} \oint \cdots \oint G\left(z_{1}, \ldots, z_{k}\right) \\
& \times q^{\frac{x}{2} \sum_{i=0}^{k} z_{i}} \frac{\Delta\left(z_{1}^{2}, \ldots, z_{k}^{2}\right)^{2} \prod_{i=1}^{k} z_{i}}{\prod_{i=1}^{k} z_{i}^{2 k}} \mathrm{~d} z_{1} \cdots \mathrm{d} z_{k},
\end{aligned}
$$

we obtain the formula of the Conjecture 2.2, i.e.

$$
\sum_{P \in \mathcal{P}_{2 g+1, q}} L\left(\frac{1}{2}, \chi_{P}\right)^{k}=\sum_{P \in \mathcal{P}_{2 g+1, q}} Q_{k}\left(\log _{q}|P|\right)(1+o(1))
$$

\section{Some conjectural formulae for moments of $L$-functions associated with $\chi_{P}$}

We use Conjecture 2.2 to obtain explicit conjectural values for several moments of quadratic Dirichlet $L$-functions associated with $\chi_{P}$ over function fields. 


\subsection{First moment}

We will use Conjecture 2.2 when $k=1$ to compute the first moment of our family of $L$-functions, then compare the result with that of Andrade and Keating proved in [3]. For $k=1$, the formula in Conjecture 2.2 gives

$$
\sum_{P \in \mathcal{P}_{2 g+1, q}} L\left(\frac{1}{2}, \chi_{P}\right)=\sum_{P \in \mathcal{P}_{2 g+1, q}} Q_{1}\left(\log _{q}|P|\right)(1+o(1)),
$$

where $Q_{1}(x)$ is polynomial of degree 1 . From the contour integral formula for $Q_{k}(x)$ in (2.14), we have

$$
Q_{1}(x)=\frac{1}{\pi i} \oint \frac{G\left(z_{1}\right) \Delta\left(z_{1}^{2}\right)^{2}}{z_{1}} q^{\frac{x}{2} z_{1}} \mathrm{~d} z_{1},
$$

where

$$
G\left(z_{1}\right)=A\left(\frac{1}{2} ; z_{1}\right) \mathcal{X}\left(\frac{1}{2}+z_{1}\right)^{-\frac{1}{2}} \zeta_{A}\left(1+2 z_{1}\right) .
$$

Recall that, the Vandermonde determinant is defined

$$
\Delta\left(z_{1}, \ldots, z_{k}\right)=\prod_{1 \leq i<j \leq k}\left(z_{j}-z_{i}\right)
$$

which for $k=1$ is equal to

$$
\Delta\left(z_{1}^{2}\right)^{2}=1
$$

and

$$
\mathcal{X}\left(\frac{1}{2}+z_{1}\right)^{-\frac{1}{2}}=q^{-z_{1} / 2} .
$$

Therefore, (4.2) becomes

$$
Q_{1}(x)=\frac{1}{\pi i} \oint \frac{A\left(\frac{1}{2} ; z_{1}\right) \zeta_{A}\left(1+2 z_{1}\right)}{z_{1}} q^{\frac{x-1}{2} z_{1}} \mathrm{~d} z_{1},
$$

with

$$
\begin{aligned}
A\left(\frac{1}{2} ; z_{1}\right)= & \prod_{\substack{P \text { monic } \\
\text { irreducible }}}\left(1-\frac{1}{|P|^{1+2 z_{1}}}\right) \\
& \times \frac{1}{2}\left(\left(1-\frac{1}{|P|^{1 / 2+z_{1}}}\right)^{-1}+\left(1+\frac{1}{|P|^{1 / 2+z_{1}}}\right)^{-1}\right) .
\end{aligned}
$$

In order to compute the integral in (4.7) where the contour is a small circle around the origin, we need to locate the poles of the integrand. So let

$$
f\left(z_{1}\right)=\frac{A\left(\frac{1}{2} ; z_{1}\right) \zeta_{A}\left(1+2 z_{1}\right)}{z_{1}} q^{\frac{x-1}{2} z_{1}}
$$

note that the zeta-function $\zeta_{A}\left(1+2 z_{1}\right)$ has a simple pole at $z_{1}=0$, which means that $f\left(z_{1}\right)$ has a pole of order 2 at $z_{1}=0$. We compute the residue by expand $f\left(z_{1}\right)$ as a 
Laurent series and consider the coefficient of $z_{1}^{-1}$. Expanding the numerator of $f\left(z_{1}\right)$ around $z_{1}=0$, we have

(1)

$$
A\left(\frac{1}{2} ; z_{1}\right)=A\left(\frac{1}{2} ; 0\right)+A^{\prime}\left(\frac{1}{2} ; 0\right) z_{1}+\frac{1}{2} A^{\prime \prime}\left(\frac{1}{2} ; 0\right) z_{1}^{2}+\cdots
$$

(2)

$$
\zeta_{A}\left(1+2 z_{1}\right)=\frac{1}{2 \log q} \frac{1}{z_{1}}+\frac{1}{2}+\frac{1}{6}(\log q) z_{1}-\frac{1}{90}(\log q)^{3} z_{1}^{3}+\cdots
$$

(3)

$$
q^{\frac{x-1}{2} z_{1}}=1+\frac{1}{2}(x-1)(\log q) z_{1}+\frac{1}{8}(x-1)^{2}(\log q)^{2} z_{1}^{2}+\cdots
$$

Hence, $f\left(z_{1}\right)$ can be written as follows:

$$
\begin{aligned}
f\left(z_{1}\right)= & \left(A\left(\frac{1}{2} ; 0\right) \frac{1}{z_{1}}+A^{\prime}\left(\frac{1}{2} ; 0\right)+\frac{1}{2} A^{\prime \prime}\left(\frac{1}{2} ; 0\right) z_{1}+\cdots\right) \\
& \times\left(\frac{1}{2 \log q} \frac{1}{z_{1}}+\frac{1}{2}+\frac{1}{6}(\log q) z_{1}-\frac{1}{90}(\log q)^{3} z_{1}^{3}+\cdots\right) \\
& \times\left(1-\frac{1}{2}(\log q) z_{1}+\frac{1}{8}(\log q)^{2} z_{1}^{2}+\cdots\right) \\
& \times\left(1+\frac{1}{2}(\log q) x z_{1}+\frac{1}{8}(\log q)^{2} x^{2} z_{1}^{2}+\cdots\right) .
\end{aligned}
$$

Considering the coefficient of $z_{1}^{-1}$, we have

$$
\operatorname{Res}_{z_{1}=0} f\left(z_{1}\right)=\frac{1}{4}(1+x) A\left(\frac{1}{2} ; 0\right)+\frac{1}{2 \log q} A^{\prime}\left(\frac{1}{2} ; z_{1}\right) .
$$
have

After straightforward calculations, using the definition for $A_{k}\left(\frac{1}{2}, z_{1}, \ldots, z_{k}\right)$, we

$$
A\left(\frac{1}{2} ; z_{1}\right)=1 \text { and } A^{\prime}\left(\frac{1}{2} ; z_{1}\right)=0
$$

and so

$$
\underset{z_{1}=0}{\operatorname{Res}} f\left(z_{1}\right)=\frac{1}{4}(1+x) .
$$

Hence, we have

$$
\begin{aligned}
Q_{1}(x) & =\frac{1}{4 \pi i}(1+x) \oint 1 \mathrm{~d} z_{1} \\
& =\frac{1}{2}(1+x) .
\end{aligned}
$$


Finally, we can write the first moment as follows:

$$
\begin{aligned}
\sum_{P \in \mathcal{P}_{2 g+1, q} L\left(\frac{1}{2}, \chi_{P}\right)} & =\sum_{P \in \mathcal{P}_{2 g+1, q}} Q_{1}\left(\log _{q}|P|\right)(1+o(1)) \\
& =\sum_{P \in \mathcal{P}_{2 g+1, q}} \frac{1}{2}\left(1+\log _{q}|P|\right)(1+o(1)) \\
& =\frac{|P|}{2 \log _{q}|P|}\left(1+\log _{q}|P|\right)+o(|P|) .
\end{aligned}
$$

If we compare Theorem 2.4 of [3] with the conjecture, we can see that the main term and the principal lower-order terms are the same. In other words, Theorem 2.4 of [3] proves our conjecture with an error $O\left(|P|^{3 / 4+\epsilon}\right)$. In the next two sections, we use our conjecture to determine the asymptotic of the second and third moments of our family of $L$-functions, and it can be seen that the polynomials $Q_{2}(x)$ and $Q_{3}(x)$ in (4.22) and (4.25) are similar to ones in [4].

\subsection{Second moment}

For $k=2$, the conjecture 2.2 gives

$$
\sum_{P \in \mathcal{P}_{2 g+1, q}} L\left(\frac{1}{2}, \chi_{P}\right)^{2}=\sum_{P \in \mathcal{P}_{2 g+1, q}} Q_{2}\left(\log _{q}|P|\right)(1+o(1)),
$$

where $Q_{2}(x)$ is a polynomial of degree 3, given by

$$
Q_{2}(x)=\frac{-1}{2 \pi^{2}} \oint \oint \frac{G\left(z_{1}, z_{2}\right) \Delta\left(z_{1}^{2}, z_{2}^{2}\right)^{2}}{z_{1}^{3} z_{2}^{3}} q^{\frac{x}{2}\left(z_{1}+z_{2}\right)} \mathrm{d} z_{1} \mathrm{~d} z_{2},
$$

with

$$
\begin{aligned}
& G\left(z_{1}, z_{2}\right)= A\left(\frac{1}{2} ; z_{1}, z_{2}\right) \mathcal{X}\left(\frac{1}{2}+z_{1}\right)^{-\frac{1}{2}} \mathcal{X}\left(\frac{1}{2}+z_{2}\right)^{-\frac{1}{2}} \\
& \times \zeta_{A}\left(1+2 z_{1}\right) \zeta_{A}\left(1+z_{1}+z_{2}\right) \zeta_{A}\left(1+2 z_{2}\right), \\
& \mathcal{X}\left(\frac{1}{2}+z_{1}\right)^{-\frac{1}{2}} \mathcal{X}\left(\frac{1}{2}+z_{2}\right)^{-\frac{1}{2}}=q^{-\frac{1}{2}\left(z_{1}+z_{2}\right)},
\end{aligned}
$$

and

$$
\Delta\left(z_{1}^{2}, z_{2}^{2}\right)^{2}=\left(z_{2}^{2}-z_{1}^{2}\right)^{2}
$$

If

$$
\begin{aligned}
f\left(z_{1}, z_{2}\right)= & \frac{A\left(\frac{1}{2} ; z_{1}, z_{2}\right) \zeta_{A}\left(1+2 z_{1}\right) \zeta_{A}\left(1+z_{1}+z_{2}\right) \zeta_{A}\left(1+2 z_{2}\right)\left(z_{2}^{2}-z_{1}^{2}\right)^{2}}{z_{1}^{3} z_{2}^{3}} \\
& \times q^{\frac{x-1}{2}\left(z_{1}+z_{2}\right)},
\end{aligned}
$$


then we have

$$
\begin{aligned}
Q_{2}(x)= & \frac{-1}{2 \pi^{2}} \oint \oint f\left(z_{1}, z_{2}\right) \mathrm{d} z_{1} \mathrm{~d} z_{2} \\
= & \frac{1}{24 \log ^{3}(q)}\left(\left(x^{3}+6 x^{2}+11 x+6\right) A(1 / 2 ; 0,0) \log ^{3}(q)\right. \\
& +\left(3 x^{2}+12 x+11\right) \\
& \times \log ^{2}(q)\left(A_{1}\left(\frac{1}{2} ; 0,0\right)+A_{2}\left(\frac{1}{2} ; 0,0\right)\right)+12(2+x) \log (q) A_{12}\left(\frac{1}{2} ; 0,0\right) \\
& \left.-2\left(A_{222}\left(\frac{1}{2} ; 0,0\right)-3 A_{122}\left(\frac{1}{2} ; 0,0\right)-3 A_{112}\left(\frac{1}{2} ; 0,0\right)+A_{111}\left(\frac{1}{2} ; 0,0\right)\right)\right),
\end{aligned}
$$

where $A_{j}$ is the partial derivative, evaluates at zero, of the function $A\left(\frac{1}{2} ; z_{1}, \cdots, z_{k}\right)$ with respect to $j$ th variable, with indices denoting higher derivatives, i.e.

$$
A_{122}\left(\frac{1}{2} ; 0, \ldots, 0\right)=\left.\frac{\partial}{\partial z_{1}} \frac{\partial^{2}}{\partial z_{2}^{2}} A\left(\frac{1}{2} ; z_{1}, \ldots, z_{k}\right)\right|_{z_{1}=z_{2}=\cdots=z_{k}=0} .
$$

Hence, we can write the leading order asymptotic for the second moment for the family of $L$-function when $g \rightarrow \infty$ as

$$
\sum_{P \in \mathcal{P}_{2 g+1, q}} L\left(\frac{1}{2}, \chi_{P}\right)^{2} \sim \frac{1}{24 \zeta_{A}(2)}|P|\left(\log _{q}|P|\right)^{2}
$$

Comparing with Andrade and Keating result (Theorem 2.5 of [3]), we see that their theorem proves our conjecture with an error $O\left(|P| \log _{q}|P|\right)$.

\subsection{Third moment}

For the third moment, Conjecture 2.2 states that

$$
\sum_{P \in \mathcal{P}_{2 g+1, q}} L\left(\frac{1}{2}, \chi_{P}\right)^{3}=\sum_{P \in \mathcal{P}_{2 g+1, q}} Q_{3}\left(\log _{q}|P|\right)(1+o(1)),
$$

where $Q_{3}(x)$ is a polynomial of degree 3 .

Thus, with the help of the symbolic manipulation software Mathematica, we compute the triple contour integral and obtain

$$
\begin{aligned}
Q_{3}(x) & =\frac{1}{8640 \log ^{6}(q)}\left(3(x+3)^{2}\left(x^{4}+12 x^{3}+49 x^{2}+78 x+40\right) A(0,0,0) \log ^{6}(q)\right. \\
& +4\left(3 x^{5}+45 x^{4}+260 x^{3}+720 x^{2}+949 x+471\right)\left(A_{3}(0,0,0)+A_{2}(0,0,0)\right. \\
+ & \left.A_{1}(0,0,0)\right) \log ^{5}(q)+4\left(15 x^{4}+180 x^{3}+780 x^{2}+1440 x+949\right)\left(A_{23}(0,0,0)\right. \\
+ & \left.A_{13}(0,0,0)+A_{12}(0,0,0)\right) \log ^{4}(q)-10\left(x^{3}+9 x^{2}+26 x+24\right)\left(2 A_{333}(0,0,0)\right.
\end{aligned}
$$




$$
\begin{gathered}
-3 A_{233}(0,0,0)-3 A_{223}(0,0,0) \\
+2 A_{222}(0,0,0)-3 A_{133}(0,0,0)-36 A_{123}(0,0,0) \\
\left.-3 A_{122}(0,0,0)-3 A_{113}(0,0,0)-3 A_{112}(0,0,0)+2 A_{111}(0,0,0)\right) \log ^{3}(q) \\
-20\left(3 x^{2}+18 x+26\right)\left(A_{2333}(0,0,0)+A_{2223}(0,0,0)\right. \\
+A_{1333}(0,0,0)-6 A_{1233}(0,0,0) \\
-6 A_{1223}(0,0,0)+A_{1222}(0,0,0)-6 A_{1123}(0,0,0) \\
\left.+A_{1113}(0,0,0)+A_{1112}(0,0,0)\right) \\
\times \log ^{2}(q)+6(x+3)\left(2 A_{33333}(0,0,0)-5 A_{23333}(0,0,0)\right. \\
-10 A_{22333}(0,0,0)-10 A_{22233}(0,0,0) \\
+5 A_{22223}(0,0,0)+2 A_{22222}(0,0,0)-5 A_{13333}(0,0,0) \\
+60 A_{12233}(0,0,0)-5 A_{12222}(0,0,0) \\
-10 A_{11333}(0,0,0)+60 A_{11233}(0,0,0) \\
+60 A_{11223}(0,0,0)-10 A_{11222}(0,0,0) \\
-10 A_{11133}(0,0,0)-10 A_{11122}(0,0,0) \\
\left.\left.+30 A_{111123}(0,0,0)+3 A_{111113}(0,0,0)+3 A_{11112}(0,0,0)\right)\right) \\
+30 A_{112333}(0,0,0)+30 A_{112223}(0,0,0) \\
-20 A_{111333}(0,0,0)+30 A_{111233}(0,0,0) \\
+30 A_{111223}(0,0,0)-20 A_{111222}(0,0,0) \\
+3 A_{23333}(0,0,0)-20 A_{222333}(0,0,0)+3 A_{222223}(0,0,0) \\
+3 A_{13333}(0,0,0)-30 A_{123333}(0,0,0) \\
\left.+5,0,0)-5 A_{11112}(0,0,0)+2 A_{11111}(0,0,0)\right) \log (q) \\
\end{gathered}
$$

where $A\left(\frac{1}{2} ; z_{1}, z_{2}, z_{3}\right)$ is defined in Lemma 3.4. Hence, the leading order asymptotic for the third moment for our family of $L$-functions is given by

$$
\sum_{P \in \mathcal{P}_{2 g+1, q}} L\left(\frac{1}{2}, \chi_{P}\right)^{3} \sim \frac{1}{2880}|P| A\left(\frac{1}{2} ; 0,0,0\right)\left(\log _{q}|P|\right)^{5}
$$

where

$$
A\left(\frac{1}{2} ; 0,0,0\right)=\prod_{\substack{P \text { monic } \\ \text { irreducible }}}\left(1-\frac{6|P|^{2}-8|P|+3}{|P|^{4}}\right) .
$$




\subsection{Leading order for general $k$}

The main aim in this section is to obtain a conjecture for the leading order asymptotics of the moments for a general integer $k$. The calculations presented here are based on the calculations first presented in $[1,26]$. To obtain the main formula we need the following lemma.

Lemma 4.1 Let $F$ be a symmetric function of $k$ variables, regular near $(0, \cdots, 0)$ and $f(s)$ has a simple pole of residue 1 at $s=0$ and analytic in a neighbourhood of $s=0$. Let

$$
\begin{aligned}
K\left(|P| ; w_{1}, \ldots, w_{k}\right)= & \sum_{\varepsilon_{i}= \pm 1} e^{\frac{1}{2} \log |P| \sum_{i=1}^{k} \varepsilon_{i} w_{i}} F\left(\varepsilon_{1} w_{1}, \ldots, \varepsilon_{k} w_{k}\right) \\
& \times \prod_{1 \leq i \leq j \leq k} f\left(\varepsilon_{i} w_{i}+\varepsilon_{j} w_{j}\right)
\end{aligned}
$$

and define $I(|P|, k ; w=0)$ to be the value of $K$ when $w_{1}, \ldots, w_{k}=0$. We have that

$$
I(|P|, k ; 0) \sim\left(\frac{1}{2} \log |P|\right)^{k(k+1) / 2} F(0, \ldots, 0) 2^{k(k+1) / 2} \cdot\left(\prod_{i=1}^{k} \frac{i !}{(2 i) !}\right)
$$

Proof See Lemma 5 in [4].

We are in a position to obtain the desired formula, from (3.55) recall that

$$
\begin{aligned}
& \sum_{P \in \mathcal{P}_{2 g+1, q}} \prod_{i=1}^{k} L\left(\frac{1}{2}+\alpha_{i}, \chi_{P}\right) \\
& =\sum_{P \in \mathcal{P}_{2 g+1, q}} \prod_{i=1}^{k} \frac{|P|^{-\frac{1}{2} \sum_{i=0}^{k} \alpha_{i}} \mathcal{X}\left(\frac{1}{2}+\alpha_{i}\right)^{\frac{1}{2}}}{(\log q)^{k(k+1) / 2}} \sum_{\varepsilon_{i}= \pm 1} K\left(\varepsilon_{1} \alpha_{1}, \ldots, \varepsilon_{k} \alpha_{k}\right)(1+o(1)),
\end{aligned}
$$

where

$$
\begin{aligned}
& K\left(\varepsilon_{1} \alpha_{1}, \ldots, \varepsilon_{k} \alpha_{k}\right) \\
& =\sum_{\varepsilon_{i}= \pm 1} \prod_{i=1}^{k} \mathcal{X}\left(\frac{1}{2}+\varepsilon_{i} \alpha_{i}\right)^{-\frac{1}{2}} A_{k}\left(\frac{1}{2} ; \alpha_{1}, \ldots, \alpha_{k}\right)|P|^{\frac{1}{2} \sum_{i=0}^{k} \varepsilon_{i} \alpha_{i}} \\
& \quad \times \prod_{1 \leq i<j \leq k} \zeta_{A}\left(1+\varepsilon_{i} \alpha_{i}+\varepsilon_{j} \alpha_{j}\right)(\log q) .
\end{aligned}
$$


Applying the above Lemma with

$$
\begin{aligned}
f(s) & =\zeta_{A}(1+s) \log q, \\
F\left(w_{1}, \ldots, w_{k}\right) & =\prod_{i=1}^{k} \mathcal{X}\left(\frac{1}{2}+\alpha_{i}\right)^{-\frac{1}{2}} A_{k}\left(\frac{1}{2} ; w_{1}, \ldots, w_{k}\right), \\
K\left(|P| ; w_{1}, \ldots, w_{k}\right)= & \sum_{\varepsilon_{i}= \pm 1}|P|^{\frac{1}{2} \sum_{i=1}^{k} \varepsilon_{i} w_{i}} F\left(\varepsilon_{1} w_{1}, \ldots, \varepsilon_{k} w_{k}\right) \\
& \times \prod_{1 \leq i \leq j \leq k} f\left(\varepsilon_{i} w_{i}+\varepsilon_{j} w_{j}\right),
\end{aligned}
$$

and letting $\alpha_{1}, \ldots, \alpha_{k} \rightarrow 0$, we obtain

$$
\begin{gathered}
\sum_{P \in \mathcal{P}_{2 g+1, q}} L\left(\frac{1}{2}, \chi_{P}\right)^{k} \sim \sum_{P \in \mathcal{P}_{2 g+1, q}} \frac{1}{(\log q)^{k(k+1) / 2}}\left(\frac{1}{2} \log |P|\right)^{\frac{k(k+1)}{2}} \\
\times A\left(\frac{1}{2} ; 0, \ldots, 0\right) 2^{\frac{k(k+1)}{2}} \prod_{i=1}^{k} \frac{i !}{(2 i) !}
\end{gathered}
$$

as $g \rightarrow \infty$. Summing over $P$, we get that

$$
\begin{aligned}
& \sum_{P \in \mathcal{P}_{2 g+1, q}} L\left(\frac{1}{2} ; \chi_{P}\right)^{k} \sim \sum_{P \in \mathcal{P}_{2 g+1, q}}\left(\log _{q}|P|\right)^{\frac{k(k+1)}{2}} A_{k}\left(\frac{1}{2} ; 0, \ldots, 0\right) \prod_{i=1}^{k} \frac{i !}{(2 i) !} \\
& =|P|\left(\log _{q}|P|\right)^{\frac{k(k+1)}{2}-1} A_{k}\left(\frac{1}{2} ; 0, \ldots, 0\right) \prod_{i=1}^{k} \frac{i !}{(2 i) !} .
\end{aligned}
$$

Hence, we have proved the following.

Theorem 4.2 Conditional on Conjecture 2.2, we have that as $g \rightarrow \infty$, the following holds:

$$
\sum_{P \in \mathcal{P}_{2 g+1, q}} L\left(\frac{1}{2}, \chi_{P}\right)^{k} \sim|P|\left(\log _{q}|P|\right)^{\frac{k(k+1)}{2}-1} A\left(\frac{1}{2} ; 0, \ldots, 0\right) \prod_{i=1}^{k} \frac{i !}{(2 i) !}
$$

\subsubsection{Some conjectural values for leading order asymptotic for the moments of $L\left(s, \chi_{P}\right)$}

We end this section by writing the asymptotic formula for the fourth and the fifth moment for our family of $L$-functions. Theorem 4.2 implies that the leading order for 
the fourth moment can be written as follows:

$$
\begin{aligned}
& \sum_{P \in \mathcal{P}_{2 g+1, q}} L\left(\frac{1}{2}, \chi_{P}\right)^{4} \sim|P|\left(\log _{q}|P|\right)^{9} A\left(\frac{1}{2} ; 0,0,0,0\right) \prod_{i=1}^{4} \frac{i !}{(2 i) !} \\
& =\frac{1}{4838400}|P|\left(\log _{q}|P|\right)^{9} A\left(\frac{1}{2} ; 0,0,0,0\right),
\end{aligned}
$$

where

$$
\begin{aligned}
& A\left(\frac{1}{2} ; 0,0,0,0\right) \\
& \quad=\prod_{\substack{P \text { monic } \\
\text { irreducible }}}\left(1-\frac{20|P|^{6}-64|P|^{5}+90|P|^{4}-64|P|^{3}+20|P|^{2}-1}{|P|^{8}}\right),
\end{aligned}
$$

and the leading order for the fifth moment is

$$
\begin{aligned}
& \sum_{P \in \mathcal{P}_{2 g+1, q}} L\left(\frac{1}{2}, \chi_{P}\right)^{5} \\
& \sim|P|\left(\log _{q}|P|\right)^{14} A\left(\frac{1}{2} ; 0,0,0,0,0\right) \prod_{i=1}^{5} \frac{i !}{(2 i) !} \\
& =\frac{1}{146313216000}|P|\left(\log _{q}|P|\right)^{14} \prod_{\substack{P \text { monic } \\
\text { irreducible }}}\left(1-\frac{h(|P|)}{|P|^{12}}\right) .
\end{aligned}
$$

with

$$
\begin{aligned}
h(x)= & 50 x^{10}-280 x^{9}+765 x^{8}-1248 x^{7}+1260 x^{6}-720 x^{5} \\
& +105 x^{4}+160 x^{3}-126 x^{2}+40 x-5 .
\end{aligned}
$$

\section{Ratios conjecture for $L$-functions over function fields}

The main aim of this section is to obtain a conjectural asymptotic formula for

$$
\sum_{P \in \mathcal{P}_{2 g+1, q}} \frac{\prod_{k=1}^{K} L\left(\frac{1}{2}+\alpha_{k}, \chi_{P}\right)}{\prod_{q=1}^{Q} L\left(\frac{1}{2}+\gamma_{q}, \chi_{P}\right)},
$$

where $\mathcal{P}_{2 g+1, q}=\left\{P\right.$ monic, $P$ irreducible, $\left.\operatorname{deg}(P)=2 g+1, P \in \mathbb{F}_{q}[T]\right\}$, and $\mathfrak{P}=\left\{L\left(s, \chi_{P}\right): P \in \mathcal{P}_{2 g+1, q}\right\}$. We adapt the original recipe of Conrey, Farmer and Zirnbauer [8] for this family of $L$-functions. 
The idea is to replace the $L$-functions in the numerator by their "approximate" functional equation:

$$
L\left(s, \chi_{P}\right)=\sum_{\substack{n \text { monic } \\ \operatorname{deg}(n) \leq g}} \frac{\chi_{P}(n)}{|n|^{s}}+\mathcal{X}_{P}(s) \sum_{\substack{n \text { monic } \\ \operatorname{deg}(n) \leq g-1}} \frac{\chi_{P}(n)}{|n|^{1-s}}
$$

and expand the $L$-functions in the denominator into the series

$$
\frac{1}{L\left(s, \chi_{P}\right)}=\prod_{\substack{P \text { monic } \\ \text { irreducible }}}\left(1-\frac{\chi_{P}(P)}{|P|^{s}}\right)=\sum_{n \text { monic }} \frac{\mu(n) \chi_{P}(n)}{|n|^{s}}
$$

where $\mu(n)$ and $\chi_{P}(n)$ are defined in Sect. 2.

As in the previous section, we apply the recipe to the quantity

$$
\sum_{P \in \mathcal{P}_{2 g+1, q}} \frac{\prod_{k=1}^{K} Z_{\mathcal{L}}\left(\frac{1}{2}+\alpha_{k}, \chi_{P}\right)}{\prod_{q=1}^{Q} L\left(\frac{1}{2}+\gamma_{q}, \chi_{P}\right)}
$$

where $Z_{\mathcal{L}}\left(s, \chi_{P}\right)$ is defined in (3.7) with "approximate" functional equation given by (3.11). Now expanding the denominator, we get

$$
\begin{aligned}
\sum_{P \in \mathcal{P}_{2 g+1, q}} & \frac{\prod_{k=1}^{K} Z_{\mathcal{L}}\left(\frac{1}{2}+\alpha_{k}, \chi_{P}\right)}{\prod_{q=1}^{Q} L\left(\frac{1}{2}+\gamma_{q}, \chi_{P}\right)} \\
\quad= & \sum_{P \in \mathcal{P}_{2 g+1, q}} \prod_{k=1}^{K} Z_{\mathcal{L}}\left(\frac{1}{2}+\alpha_{k}, \chi_{P}\right) \sum_{\substack{h_{1}, \ldots, h_{Q} \\
h_{q} \text { monic }}} \frac{\mu\left(h_{1}\right) \cdots \mu\left(h_{Q}\right) \chi_{P}\left(h_{1} \cdots h_{Q}\right)}{\left|h_{1}\right|^{\frac{1}{2}+\gamma_{1}} \cdots\left|h_{Q}\right|^{\frac{1}{2}+\gamma_{Q}}} .
\end{aligned}
$$

Making use of the "approximate" functional equation (5.2), we have

$$
\begin{aligned}
& \prod_{k=1}^{K} Z_{\mathcal{L}}\left(\frac{1}{2}+\alpha_{k}, \chi_{P}\right) \\
& \quad=\sum_{\varepsilon_{k} \in\{-1,1\}^{K}} \prod_{k=1}^{K} \mathcal{X}_{P}\left(\frac{1}{2}+\varepsilon_{k} \alpha_{k}\right)^{-\frac{1}{2}} \sum_{\substack{m_{1}, \ldots, m_{K} \\
m_{i} \text { monic }}} \frac{\chi_{P}\left(m_{1} \cdots m_{K}\right)}{\left|m_{1}\right|^{\frac{1}{2}+\varepsilon_{1} \alpha_{1}} \cdots\left|m_{K}\right|^{\frac{1}{2}+\varepsilon_{K} \alpha_{K}}},
\end{aligned}
$$


so we can write (5.5) as follows:

$$
\begin{aligned}
\sum_{P \in \mathcal{P}_{2 g+1, q}} & \frac{\prod_{k=1}^{K} Z_{\mathcal{L}}\left(\frac{1}{2}+\alpha_{k}, \chi_{P}\right)}{\prod_{q=1}^{Q} L\left(\frac{1}{2}+\gamma_{q}, \chi_{P}\right)} \\
= & \sum_{P \in \mathcal{P}_{2 g+1, q}} \sum_{\varepsilon_{k} \in\{-1,1\}^{K}} \prod_{k=1}^{K} \mathcal{X}_{P}\left(\frac{1}{2}+\varepsilon_{k} \alpha_{k}\right)^{-\frac{1}{2}} \\
& \times \sum_{\substack{m_{1}, \cdots, m_{K} \\
h_{1}, \cdots, h_{Q} \\
m_{i}, h_{j} \text { monic }}} \frac{\prod_{q=1}^{Q} \mu\left(h_{q}\right) \chi_{P}\left(\prod_{k=1}^{K} m_{k} \prod_{q=1}^{Q} h_{q}\right)}{\prod_{k=1}^{K}\left|m_{k}\right|^{\frac{1}{2}+\varepsilon_{k} \alpha_{k}} \prod_{q=1}^{Q}\left|h_{q}\right|^{\frac{1}{2}+\gamma_{q}}} .
\end{aligned}
$$

Following the recipe, we replace each summand by its expected value when averaged over primes $P \in \mathcal{P}_{2 g+1, q}$, in other words, we have that

$$
\begin{aligned}
& \lim _{\operatorname{deg}(P) \rightarrow \infty}\left(\frac{1}{\# \mathcal{P}_{2 g+1, q}} \sum_{P \in \mathcal{P}_{2 g+1, q}} \sum_{\varepsilon_{k} \in\{-1,1\}^{K}} \prod_{k=1}^{K} \mathcal{X}_{P}\left(\frac{1}{2}+\varepsilon_{k} \alpha_{k}\right)^{-\frac{1}{2}}\right. \\
& \left.\times \sum_{\substack{m_{1}, \ldots, m_{K} \\
h_{1}, \ldots, h_{Q} \\
m_{i}, h_{j} \text { monic }}} \frac{\prod_{q=1}^{Q} \mu\left(h_{q}\right) \chi_{P}\left(\prod_{k=1}^{K} m_{k} \prod_{q=1}^{Q} h_{q}\right)}{\prod_{k=1}^{K}\left|m_{k}\right|^{\frac{1}{2}+\varepsilon_{k} \alpha_{k}} \prod_{q=1}^{Q}\left|h_{q}\right|^{\frac{1}{2}+\gamma_{q}}}\right) \\
& =\sum_{\varepsilon_{k} \in\{-1,1\}^{K}} \prod_{k=1}^{K} \mathcal{X}_{P}\left(\frac{1}{2}+\varepsilon_{k} \alpha_{k}\right)^{-\frac{1}{2}} \\
& \quad \sum_{\substack{m_{1}, \ldots, m_{K} \\
h_{1}, \ldots, h_{Q} \\
m_{i}, h_{j} \text { monic }}} \frac{\prod_{q=1}^{Q} \mu\left(h_{q}\right) \delta\left(\prod_{k=1}^{K} m_{k} \prod_{q=1}^{Q} h_{q}\right)}{\prod_{k=1}^{K}\left|m_{k}\right|^{\frac{1}{2}+\varepsilon_{k} \alpha_{k}} \prod_{q=1}^{Q}\left|h_{q}\right|^{\frac{1}{2}+\gamma_{q}}}
\end{aligned}
$$

where $\delta(n)=1$ if $n$ is a square and 0 otherwise.

Next we factor out the zeta-function factors. Note that, the main difficulty here is to identify and factor out the appropriate zeta-functions factors that contribute to poles and zeros. With the same notation used in [1], we define the following series:

$$
G_{\mathfrak{P}}(\alpha ; \gamma)=\sum_{\substack{m_{1}, \ldots, m_{K} \\ h_{1}, \ldots, h_{Q} \\ m_{i}, h_{j} \text { monic }}} \frac{\prod_{q=1}^{Q} \mu\left(h_{q}\right) \delta\left(\prod_{k=1}^{K} m_{k} \prod_{q=1}^{Q} h_{q}\right)}{\prod_{k=1}^{K}\left|m_{k}\right|^{\frac{1}{2}+\varepsilon_{k} \alpha_{k}} \prod_{q=1}^{Q}\left|h_{q}\right|^{\frac{1}{2}+\gamma_{q}}}
$$


If $m_{k}=\prod_{P} P^{a_{k}}$ and $h_{q}=\prod_{P} P^{c_{q}}$, then we can write $G_{\mathfrak{P}}(\alpha ; \gamma)$ as a convergent Euler product provided that $\mathfrak{R}\left(\alpha_{k}\right)>0$ and $\mathfrak{R}\left(\gamma_{q}\right)>0$,

$$
\begin{aligned}
& G_{\mathfrak{P}}(\alpha ; \gamma) \\
& \quad=\prod_{\substack{P \text { monic } \\
\text { irreducible }}}\left(1+\sum_{0<\sum_{k} a_{k}+\sum_{q} c_{q} \text { is even }} \frac{\prod_{q=1}^{Q} \mu\left(P^{c_{q}}\right)}{|P|^{\sum_{k} a_{k}\left(\frac{1}{2}+\alpha_{k}\right)+\sum_{q} c_{q}\left(\frac{1}{2}+\gamma_{q}\right)}}\right) .
\end{aligned}
$$

We now write $G_{\mathfrak{P}}$ in terms of the zeta-function of $\mathbb{F}_{q}[T]$. First, we express the contribution of all poles and zeros of (5.10) in terms of $\zeta_{A}(s)$ by rewriting the Euler product in (5.10) as follows:

$$
\begin{aligned}
G_{\mathfrak{P}}(\alpha ; \gamma)= & \prod_{\substack{P \text { monic } \\
\text { irreducible }}}\left(1+\sum_{\substack{j, k \\
j<k}} \frac{1}{|P|^{\left(\frac{1}{2}+\alpha_{j}\right)+\left(\frac{1}{2}+\alpha_{k}\right)}}+\sum_{k} \frac{1}{|P|^{\left(1+2 \alpha_{k}\right)}}\right. \\
& \left.+\sum_{\substack{r, q \\
r<q}} \frac{\mu(P)^{2}}{|P|^{\left(\frac{1}{2}+\gamma_{r}\right)+\left(\frac{1}{2}+\gamma_{q}\right)}}+\sum_{k} \sum_{q} \frac{\mu(P)}{|P|^{\left(\frac{1}{2}+\alpha_{k}\right)+\left(\frac{1}{2}+\gamma_{q}\right)}}+\cdots\right),
\end{aligned}
$$

where $\cdots$ are referring to the convergent terms. Recall that

$$
\zeta_{A}(s)=\prod_{\substack{P \text { monic } \\ \text { irreducible }}}\left(1-\frac{1}{|P|^{s}}\right)^{-1}=\prod_{\substack{P \text { monic } \\ \text { irreducible }}}\left(\sum_{j=0}^{\infty}\left(\frac{1}{|P|^{s}}\right)^{j}\right)
$$

We can see from (5.11) that the terms with $\sum_{k=1}^{K} a_{k}+\sum_{q=1}^{Q} c_{q}=2$ contribute to the poles and zeros. The poles are coming from the terms with $a_{j}=a_{k}=1,1 \leq j<k \leq$ $K, a_{k}=2,1 \leq k \leq K$, and also from the terms with $c_{r}=c_{q}=1,1 \leq r<q \leq Q$. Note that there are no poles coming from the terms with $c_{q}=2,1 \leq q \leq Q$, since $\mu\left(P^{2}\right)=0$. Moreover, the zeros come from the terms with $a_{k}=c_{q}=1$ with $1 \leq k \leq K$, and $1 \leq q \leq Q$.

From the above, we can define the function $Y_{\mathfrak{P}}(\alpha ; \gamma)$ in terms of $\zeta_{A}(s)$ by

$$
Y_{\mathfrak{P}}(\alpha ; \gamma):=\frac{\prod_{1 \leq j \leq k \leq K} \zeta_{A}\left(1+\alpha_{j}+\alpha_{k}\right) \prod_{1 \leq r \leq q \leq Q} \zeta_{A}\left(1+\gamma_{r}+\gamma_{q}\right)}{\prod_{k=1}^{k} \prod_{q=1}^{Q} \zeta_{A}\left(1+\alpha_{k}+\gamma_{q}\right)}
$$

Thus, we can factor out $Y_{\mathfrak{P}}(\alpha ; \gamma)$ from $G_{\mathfrak{P}}(\alpha ; \gamma)$, such that

$$
G_{\mathfrak{P}}(\alpha ; \gamma)=Y_{\mathfrak{P}}(\alpha ; \gamma) A_{\mathfrak{P}}(\alpha ; \gamma),
$$


where $A_{\mathfrak{P}}(\alpha ; \gamma)$ is the Euler product that converges absolutely for all of the variables in the small discs around 0 :

$$
\begin{aligned}
A_{\mathfrak{P}}(\alpha ; \gamma) & \\
= & \prod_{\substack{P \text { monic } \\
\text { irreducible }}} \frac{\prod_{1 \leq j \leq k \leq K}\left(1-\frac{1}{|P|^{1+\alpha_{j}+\alpha_{k}}}\right) \prod_{1 \leq r \leq q \leq Q}\left(1-\frac{1}{|P|^{1+\gamma_{r}+\gamma_{q}}}\right)}{\prod_{k=1}^{k} \prod_{q=1}^{Q}\left(1-\frac{1}{|P|^{1+\alpha_{k}+\gamma_{q}}}\right)} \\
& \times\left(1+\sum_{0<\sum_{k} a_{k}+\sum_{q} c_{q} \text { is even }}^{|P|^{\sum_{k} a_{k}\left(\frac{1}{2}+\alpha_{k}\right)+\sum_{q} c_{q}\left(\frac{1}{2}+\gamma_{q}\right)}}\right) .
\end{aligned}
$$

Returning to the recipe, we can conclude from (5.7), (5.9), and (5.14) that

$$
\begin{aligned}
& \sum_{P \in \mathcal{P}_{2 g+1, q}} \frac{\prod_{k=1}^{K} Z_{\mathcal{L}}\left(\frac{1}{2}+\alpha_{k}, \chi_{P}\right)}{\prod_{q=1}^{Q} L\left(\frac{1}{2}+\gamma_{q}, \chi_{P}\right)} \\
& =\sum_{P \in \mathcal{P}_{2 g+1, q}} \sum_{\varepsilon_{k} \in\{-1,1\}^{K}} \prod_{k=1}^{K} \mathcal{X}_{P}\left(\frac{1}{2}+\varepsilon_{k} \alpha_{k}\right)^{-\frac{1}{2}} Y_{\mathfrak{P}}\left(\varepsilon_{1} \alpha_{1}, \cdots, \varepsilon_{k} \alpha_{k} ; \gamma\right) \\
& \quad \times A_{\mathfrak{P}}\left(\varepsilon_{1} \alpha_{1}, \cdots, \varepsilon_{k} \alpha_{k} ; \gamma\right)+o(|P|)
\end{aligned}
$$

Now, using (3.7), we have

$$
\begin{aligned}
& \sum_{P \in \mathcal{P}_{2 g+1, q}} \frac{\prod_{k=1}^{K} L\left(\frac{1}{2}+\alpha_{k}, \chi_{P}\right)}{\prod_{q=1}^{Q} L\left(\frac{1}{2}+\gamma_{q}, \chi_{P}\right)} \\
& =\sum_{P \in \mathcal{P}_{2 g+1, q}} \sum_{\varepsilon_{k} \in\{-1,1\}^{K}} \prod_{k=1}^{K} \mathcal{X}_{P}\left(\frac{1}{2}+\alpha_{k}\right)^{\frac{1}{2}} \mathcal{X}_{P}\left(\frac{1}{2}+\alpha_{k}\right)^{-\frac{1}{2}} \\
& \quad \times Y_{\mathfrak{P}}\left(\varepsilon_{1} \alpha_{1}, \ldots, \varepsilon_{k} \alpha_{k} ; \gamma\right) A_{\mathfrak{P}}\left(\varepsilon_{1} \alpha_{1}, \ldots, \varepsilon_{k} \alpha_{k} ; \gamma\right)+o(|P|) .
\end{aligned}
$$

Remembering that

$$
\mathcal{X}_{P}(s)=|P|^{\frac{1}{2}-s} \mathcal{X}(s)
$$

with

$$
\mathcal{X}(s)=q^{-\frac{1}{2}+s},
$$

we have that

$$
\begin{aligned}
& \prod_{k=1}^{K} \mathcal{X}_{P}\left(\frac{1}{2}+\varepsilon_{k} \alpha_{k}\right)^{-\frac{1}{2}} \mathcal{X}_{P}\left(\frac{1}{2}+\varepsilon_{k} \alpha_{k}\right)^{\frac{1}{2}} \\
& \quad=|P|^{\frac{1}{2} \sum_{k=1}^{K}\left(\varepsilon_{k} \alpha_{k}-\alpha_{k}\right)} \prod_{k=1}^{K} \mathcal{X}\left(\frac{1}{2}+\frac{\alpha_{k}-\varepsilon_{k} \alpha_{k}}{2}\right) .
\end{aligned}
$$


For positive real parts of $\alpha_{k}$ and $\gamma_{q}$, we have

$$
\begin{aligned}
& \sum_{P \in \mathcal{P}_{2 g+1, q}} \frac{\prod_{k=1}^{K} L\left(\frac{1}{2}+\alpha_{k}, \chi_{P}\right)}{\prod_{q=1}^{Q} L\left(\frac{1}{2}+\gamma_{q}, \chi_{P}\right)} \\
& =\sum_{P \in \mathcal{P}_{2 g+1, q}} \sum_{\varepsilon_{k} \in\{-1,1\}^{K}}|P|^{\frac{1}{2} \sum_{k=1}^{K}\left(\varepsilon_{k} \alpha_{k}-\alpha_{k}\right)} \prod_{k=1}^{K} \mathcal{X}\left(\frac{1}{2}+\frac{\alpha_{k}-\varepsilon_{k} \alpha_{k}}{2}\right) \\
& \quad \times Y_{\mathfrak{P}}\left(\varepsilon_{1} \alpha_{1}, \ldots, \varepsilon_{k} \alpha_{k} ; \gamma\right) A_{\mathfrak{P}}\left(\varepsilon_{1} \alpha_{1}, \ldots, \varepsilon_{k} \alpha_{k} ; \gamma\right)+o(|P|) .
\end{aligned}
$$

Finally, if we let

$$
\begin{aligned}
H_{\mathfrak{P},|P|, \alpha, \gamma}(w ; \gamma)= & |P|^{\frac{1}{2} \sum_{k=1}^{k} w_{k}} \prod_{k=1}^{K} \mathcal{X}\left(\frac{1}{2}+\frac{\alpha_{k}-w_{k}}{2}\right) \\
& \times Y_{\mathfrak{P}}(w ; \gamma) A_{\mathfrak{P}}(w ; \gamma),
\end{aligned}
$$

then the conjecture may be formulated as follows:

$$
\begin{aligned}
& \sum_{P \in \mathcal{P}_{2 g+1, q}} \frac{\prod_{k=1}^{K} L\left(\frac{1}{2}+\alpha_{k}, \chi_{P}\right)}{\prod_{q=1}^{Q} L\left(\frac{1}{2}+\gamma_{q}, \chi_{P}\right)} \\
& =\sum_{P \in \mathcal{P}_{2 g+1, q}}|P|^{-\frac{1}{2} \sum_{k=1}^{K} \alpha_{k}} \sum_{\varepsilon \in\{-1,1\}^{K}} H_{\mathfrak{P},|P|, \alpha, \gamma}(\varepsilon \alpha ; \gamma)+o(|P|) .
\end{aligned}
$$

\subsection{Refinements of Conjecture}

In this section, we state the final form of our ratios conjecture. In the first part, we derive a closed form expression for the Euler product $A_{\mathfrak{P}}(\alpha ; \gamma)$, and in the second part, we express the combinatorial sum as a multiple integral.

\subsubsection{Closed form expression for $A_{\mathfrak{P}}$}

Suppose that $f(x)=1+\sum_{n=1}^{\infty} u_{n} x^{n}$, then

$$
\sum_{n \text { even }} u_{n} x^{n}=\frac{1}{2}(f(x)+f(-x)-2),
$$


and so, let

$$
\begin{aligned}
f\left(\frac{1}{|P|}\right) & =\sum_{a_{k}, c_{q}} \frac{\prod_{q=1}^{Q} \mu\left(P^{c_{q}}\right)}{|P|^{\sum_{k} a_{k}\left(\frac{1}{2}+\alpha_{k}\right)+\sum_{q} c_{q}\left(\frac{1}{2}+\gamma_{q}\right)}} \\
& =\sum_{a_{k}} \prod_{k=1}^{K} \frac{1}{|P|^{a_{k}\left(\frac{1}{2}+\alpha_{k}\right)}} \sum_{c_{q}} \prod_{q=1}^{Q} \frac{\mu\left(P^{c_{q}}\right)}{|P|^{c_{q}\left(\frac{1}{2}+\gamma_{q}\right)}} \\
& =\frac{\prod_{q=1}^{Q}\left(1-\frac{1}{|P|^{\frac{1}{2}+\gamma_{q}}}\right)}{\prod_{k=1}^{K}\left(1-\frac{1}{|P|^{\frac{1}{2}+\alpha_{k}}}\right)} .
\end{aligned}
$$

Using the above equations, we can establish the following lemma.

Lemma 5.1 We have that,

$$
\begin{aligned}
& \left.1+\sum_{\sum_{k} a_{k}+\sum_{q} c_{q} \text { even }} \frac{\prod_{q=1}^{Q} \mu\left(P^{\sum_{k} a_{k}\left(\frac{1}{2}+\alpha_{k}\right)+\sum_{q} c_{q}\left(\frac{1}{2}+\gamma_{q}\right)}\right.}{\mid \prod^{\frac{1}{2}+\gamma_{q}}}\right) \\
& \quad=\frac{1}{2}\left(\frac{\prod_{q=1}^{Q}\left(1-\frac{1}{\mid \prod^{\frac{1}{2}+\alpha_{k}}}\right)}{\prod_{k=1}^{K}\left(1-\frac{1}{|P|^{\frac{1}{2}+\gamma_{q}}}\right)}\right)
\end{aligned}
$$

The following result is a direct corollary from Lemma 5.1 and Eq. (5.15).

\section{Corollary 5.2}

$$
\begin{aligned}
A_{\mathfrak{P}}(\alpha ; \gamma)= & \prod_{\substack{P \text { monic } \\
\text { irreducible }}} \frac{\prod_{1 \leq j \leq k \leq K}\left(1-\frac{1}{|P|^{1+\alpha_{j}+\alpha_{k}}}\right) \prod_{1 \leq r \leq q \leq Q}\left(1-\frac{1}{|P|^{1+\gamma_{r}+\gamma_{q}}}\right)}{\prod_{k=1}^{k} \prod_{q=1}^{Q}\left(1-\frac{1}{|P|^{1+\alpha_{k}+\gamma_{q}}}\right)} \\
& \times\left(\frac{1}{2}\left(\frac{\prod_{q=1}^{Q}\left(1-\frac{1}{|P|^{\frac{1}{2}+\gamma_{q}}}\right)}{\prod_{k=1}^{K}\left(1-\frac{1}{|P|^{\frac{1}{2}+\alpha_{k}}}\right)}+\frac{\prod_{q=1}^{Q}\left(1+\frac{1}{|P|^{\frac{1}{2}+\gamma_{q}}}\right)}{\prod_{k=1}^{K}\left(1+\frac{1}{|P|^{\frac{1}{2}+\alpha_{k}}}\right)}\right)\right)
\end{aligned}
$$

\subsubsection{The final form of the ratios conjecture}

To obtain our final form of the Ratios Conjecture 2.3, we need the following lemma (Lemma 6.8, [8]).

Lemma 5.3 Suppose that $F(z)=F\left(z_{1}, \cdots, z_{K}\right)$ is a function of $K$ variables, which is symmetric and regular near $(0, \cdots, 0)$. Suppose further that $f(s)$ has a simple pole 
of residue 1 at $s=0$ but is otherwise analytic in $|s| \leq 1$. Let either

$$
H\left(z_{1}, \ldots, z_{K}\right)=F\left(z_{1}, \ldots, z_{K}\right) \prod_{1 \leq j \leq k \leq K} f\left(z_{j}+z_{k}\right)
$$

or

$$
H\left(z_{1}, \ldots, z_{K}\right)=F\left(z_{1}, \ldots, z_{K}\right) \prod_{1 \leq j<k \leq K} f\left(z_{j}+z_{k}\right) .
$$

If $\left|\alpha_{k}\right|<1$, then

$$
\begin{aligned}
& \sum_{\varepsilon \in\{-1,1\}^{K}} H\left(\varepsilon_{1} \alpha_{1}, \ldots, \varepsilon_{K} \alpha_{K}\right) \\
& =\frac{(-1)^{K(K-1) / 2} 2^{K}}{K !(2 \pi i)^{K}} \int_{\left|z_{i}\right|=1} \frac{H\left(z_{1}, \ldots, z_{K}\right) \Delta\left(z_{1}^{2}, \ldots, z_{K}^{2}\right)^{2} \prod_{k=1}^{K} z_{k}}{\prod_{j=1}^{K} \prod_{k=1}^{K}\left(z_{k}-\alpha_{j}\right)\left(z_{k}+\alpha_{j}\right)} \mathrm{d} z_{1} \cdots \mathrm{d} z_{K}
\end{aligned}
$$

and

$$
\begin{aligned}
& \sum_{\varepsilon \in\{-1,1\}^{K}} \operatorname{sgn}(\varepsilon) H\left(\varepsilon_{1} \alpha_{1}, \ldots, \varepsilon_{K} \alpha_{K}\right) \\
& =\frac{(-1)^{K(K-1) / 2} 2^{K}}{K !(2 \pi i)^{K}} \int_{\left|z_{i}\right|=1} \frac{H\left(z_{1}, \ldots, z_{K}\right) \Delta\left(z_{1}^{2}, \ldots, z_{K}^{2}\right)^{2} \prod_{k=1}^{K} \alpha_{k}}{\prod_{j=1}^{K} \prod_{k=1}^{K}\left(z_{k}-\alpha_{j}\right)\left(z_{k}+\alpha_{j}\right)} \mathrm{d} z_{1} \cdots \mathrm{d} z_{K} .
\end{aligned}
$$

Now, we are in a position to present the final form of the ratios conjecture 2.3.

Conjecture 5.4 Suppose that the real parts of $\alpha_{k}$ and $\gamma_{q}$ are positive. Then we have,

$$
\begin{aligned}
& \sum_{P \in \mathcal{P}_{2 g+1, q}} \frac{\prod_{k=1}^{K} L\left(\frac{1}{2}+\alpha_{k}, \chi_{P}\right)}{\prod_{q=1}^{Q} L\left(\frac{1}{2}+\gamma_{q}, \chi_{P}\right)} \\
& =\sum_{P \in \mathcal{P}_{2 g+1, q}}|P|^{-\frac{1}{2} \sum_{k=1}^{K} \alpha_{k}} \frac{(-1)^{K(K-1) / 2} 2^{K}}{K !(2 \pi i)^{K}} \\
& \quad \times \int_{\left|z_{i}\right|=1} \frac{H_{\mathfrak{P},|P|, \alpha, \gamma}\left(z_{1}, \ldots, z_{K}\right) \Delta\left(z_{1}^{2}, \ldots, z_{K}^{2}\right)^{2} \prod_{k=1}^{K} z_{k}}{\prod_{j=1}^{K} \prod_{k=1}^{K}\left(z_{k}-\alpha_{j}\right)\left(z_{k}+\alpha_{j}\right)} \cdot \mathrm{d} z_{K} \\
& \quad+o(|P|) .
\end{aligned}
$$

\section{One-level density}

In this section, we give an application of the Ratios Conjecture 2.3 for $L$-functions over function fields. We compute a smooth linear statistic, the one-level density for the family of quadratic Dirichlet $L$-functions associated with monic irreducible polynomials in $\mathbb{F}_{q}[T]$. The one-level density for the family of quadratic Dirichlet $L$-functions 
over fundamental discriminants was computed using the rations conjecture by Conrey and Snaith [15] in the number field setting and by Andrade and Keating [4] in the function field setting.

Consider

$$
R_{P}(\alpha ; \gamma)=\sum_{P \in \mathcal{P}_{2 g+1, q}} \frac{L\left(\frac{1}{2}+\alpha, \chi_{P}\right)}{L\left(\frac{1}{2}+\gamma, \chi_{P}\right)}
$$

Using the ratios conjecture as presented in the last section with one $L$-function in the numerator and one $L$-function in the denominator, we arrive at the following particular conjecture.

Conjecture 6.1 With $-\frac{1}{4}<\mathfrak{R}(\alpha)<\frac{1}{4}, \frac{1}{\log |P|} \ll \mathfrak{R}(\gamma)<\frac{1}{4}$ and $\mathfrak{I}(\alpha), \mathfrak{I}(\gamma) \ll_{\epsilon}$ $|P|^{1-\epsilon}$ for every $\epsilon>0$, we have

$$
\begin{aligned}
R_{P}(\alpha ; \gamma)= & \sum_{P \in \mathcal{P}_{2 g+1, q}} \frac{L\left(\frac{1}{2}+\alpha, \chi_{P}\right)}{L\left(\frac{1}{2}+\gamma, \chi_{P}\right)} \\
= & \sum_{P \in \mathcal{P}_{2 g+1, q}}\left(\frac{\zeta_{A}(1+2 \alpha)}{\zeta_{A}(1+\alpha+\gamma)}+|P|^{-\alpha} \mathcal{X}\left(\frac{1}{2}+\alpha\right)\right. \\
& \left.\times \frac{\zeta_{A}(1-2 \alpha)}{\zeta_{A}(1-\alpha+\gamma)}\right)+o(|P|) .
\end{aligned}
$$

To compute the one-level density, we need to have a formula for

$$
\sum_{P \in \mathcal{P}_{2 g+1, q}} \frac{L^{\prime}\left(\frac{1}{2}+r, \chi_{P}\right)}{L\left(\frac{1}{2}+r, \chi_{P}\right)}=\left.\frac{\mathrm{d}}{\mathrm{d} \alpha} R_{\mathcal{P}}(\alpha ; \gamma)\right|_{\alpha=\gamma=r}
$$

A direct calculation gives

$$
\left.\frac{\mathrm{d}}{\mathrm{d} \alpha}\left(\frac{\zeta_{A}(1+2 \alpha)}{\zeta_{A}(1+\alpha+\gamma)}\right)\right|_{\alpha=\gamma=r}=\frac{\zeta_{A}^{\prime}(1+2 r)}{\zeta_{A}(1+2 r)}
$$

and that

$$
\begin{aligned}
& \left.\frac{\mathrm{d}}{\mathrm{d} \alpha}\left(|P|^{-\alpha} \mathcal{X}\left(\frac{1}{2}+\alpha\right) \frac{\zeta_{A}(1-2 \alpha)}{\zeta_{A}(1-\alpha+\gamma)}\right)\right|_{\alpha=\gamma=r} \\
& =-(\log q)|P|^{-r} \mathcal{X}\left(\frac{1}{2}+r\right) \zeta_{A}(1-2 r) .
\end{aligned}
$$

Therefore, the ratios conjecture implies that the following result holds. 
Theorem 6.2 Assuming Conjecture 6.1, $\frac{1}{\log |P|} \ll \Re(r)<\frac{1}{4}$ and $\mathfrak{I}(r) \ll_{\epsilon}|P|^{1-\epsilon}$ for every $\epsilon>0$, we have

$$
\begin{aligned}
\sum_{P \in \mathcal{P}_{2 g+1, q}} & \frac{L^{\prime}\left(\frac{1}{2}+r, \chi_{P}\right)}{L\left(\frac{1}{2}+r, \chi_{P}\right)} \\
= & \sum_{P \in \mathcal{P}_{2 g+1, q}}\left(\frac{\zeta_{A}^{\prime}(1+2 r)}{\zeta_{A}(1+2 r)}-(\log q)|P|^{-r} \mathcal{X}\left(\frac{1}{2}+r\right)\right. \\
& \left.\times \zeta_{A}(1-2 r)\right)+o(|P|) .
\end{aligned}
$$

We have available all the necessary machinery to derive the formula for the onelevel density for the zeros of Dirichlet $L$-functions associated to quadratic characters $\chi_{P}$ with $P \in \mathcal{P}_{2 g+1, q}$, complete with lower-order terms.

Let $\gamma_{P}$ be the ordinate of a generic zero of $L\left(s, \chi_{P}\right)$ on the half-line. Since $L\left(s, \chi_{P}\right)$ is a function of $u=q^{-S}$ and periodic with period $2 \pi i / \log q$, we can restrict our analysis of the zeros for the range $-\pi i / \log q \leq \Im(s) \leq \pi i / \log q$. Consider the one-level density

$$
S_{1}(f):=\sum_{P \in \mathcal{P}_{2 g+1, q}} \sum_{\gamma_{P}} f\left(\gamma_{P}\right),
$$

where $f$ is an even $2 \pi / \log q$-periodic test functions and holomorphic.

Using Cauchy's Theorem, we have

$$
S_{1}(f)=\sum_{P \in \mathcal{P}_{2 g+1, q}} \frac{1}{2 \pi i}\left(\int_{(c)}-\int_{(1-c)}\right) \frac{L^{\prime}\left(s, \chi_{P}\right)}{L\left(s, \chi_{P}\right)} f(-i(s-1 / 2)) \mathrm{d} s
$$

where $(c)$ is the vertical line from $c-\pi i / \log q$ to $c+\pi i / \log q$ and $1 / 2+1 / \log |P|<$ $c<3 / 4$. For the integral on the (c)-line, we make the following variable change, letting $s \rightarrow c+i t$, so

$$
\frac{1}{2 \pi} \int_{-\pi / \log q}^{\pi / \log q} f(-i(i t+c-1 / 2)) \sum_{P \in \mathcal{P}_{2 g+1, q}} \frac{L^{\prime}\left(c+i t, \chi_{P}\right)}{L\left(c+i t, \chi_{P}\right)} \mathrm{d} t .
$$

Since the integrand is regular at $t=0$, we move the path of the integration to $c=1 / 2$ and replace the sum over $P$ by Theorem 6.2 to obtain

$$
\begin{aligned}
& \frac{1}{2 \pi} \int_{-\pi / \log q}^{\pi / \log q} f(t) \sum_{P \in \mathcal{P}_{2 g+1, q}}\left(\frac{\zeta_{A}^{\prime}(1+2 i t)}{\zeta_{A}(1+2 i t)}\right. \\
& \left.\quad-(\log q)|P|^{-i t} \mathcal{X}\left(\frac{1}{2}+i t\right) \zeta_{A}(1-2 i t)\right) \mathrm{d} t+o(|P|)
\end{aligned}
$$


The functional equation (2.10) implies that

$$
\frac{L^{\prime}\left(1-s, \chi_{P}\right)}{L\left(1-s, \chi_{P}\right)}=\frac{\mathcal{X}_{P}^{\prime}(s)}{\mathcal{X}_{P}(s)}-\frac{L^{\prime}\left(s, \chi_{P}\right)}{L\left(s, \chi_{P}\right)}
$$

with

$$
\frac{\mathcal{X}_{P}^{\prime}(s)}{\mathcal{X}_{P}(s)}=-\log |P|+\frac{\mathcal{X}^{\prime}(s)}{\mathcal{X}(s)} \text {. }
$$

For the integral on the $(1-c)$-line, we change variables, letting $s \rightarrow 1-s$, then use (6.11) and with the similar calculations as for the integral on the (c)-line, we obtain the following theorem.

Theorem 6.3 Assuming the ratios Conjecture 6.1, we have that

$$
\begin{aligned}
S_{1}(f)= & \sum_{P \in \mathcal{P}_{2 g+1, q}} \sum_{\gamma_{P}} f\left(\gamma_{P}\right) \\
= & \frac{1}{2 \pi} \int_{-\pi / \log q}^{\pi / \log q} f(t) \sum_{P \in \mathcal{P}_{2 g+1, q}}\left(\log |P|+\frac{\mathcal{X}^{\prime}\left(\frac{1}{2}-i t\right)}{\mathcal{X}\left(\frac{1}{2}-i t\right)}\right. \\
& \left.+2\left(\frac{\zeta_{A}^{\prime}(1+2 i t)}{\zeta_{A}(1+2 i t)}-(\log q)|P|^{-i t} \mathcal{X}\left(\frac{1}{2}+i t\right) \zeta_{A}(1-2 i t)\right)\right) \mathrm{d} t \\
& +o(|P|),
\end{aligned}
$$

where $\gamma_{P}$ is the ordinate of a generic zero of $L\left(s, \chi_{P}\right)$ and $f$ is an even and periodic suitable test function.

\subsection{The scaled one-level density}

Defining

$$
f(t)=h\left(\frac{t(2 g \log q)}{2 \pi}\right)
$$

and scaling the variable $t$ from Theorem 6.3 as

$$
\tau=\frac{t(2 g \log q)}{2 \pi}
$$


we have that

$$
\begin{aligned}
& \sum_{P \in \mathcal{P}_{2 g+1, q}} \sum_{\gamma_{P}} f\left(\gamma_{P} \frac{2 g \log q}{2 \pi}\right) \\
& =\frac{1}{2 g \log q} \int_{-g}^{g} h(\tau) \sum_{P \in \mathcal{P}_{2 g+1, q}}\left(\log |P|+\frac{\mathcal{X}^{\prime}\left(\frac{1}{2}-\frac{2 \pi i \tau}{2 g \log q}\right)}{\mathcal{X}\left(\frac{1}{2}-\frac{2 \pi i \tau}{2 g \log q}\right)}\right. \\
& \quad+2\left(\frac{\zeta_{A}^{\prime}\left(1+\frac{4 \pi i \tau}{2 g \log q}\right)}{\zeta_{A}\left(1+\frac{4 \pi i \tau}{2 g \log q}\right)}-(\log q) e^{(-2 \pi i \tau / 2 g \log q) \log |P|} \mathcal{X}\left(\frac{1}{2}+\frac{2 \pi i \tau}{2 g \log q}\right)\right. \\
& \left.\left.\quad \times \zeta_{A}\left(1-\frac{4 \pi i \tau}{2 g \log q}\right)\right)\right) d \tau+o(|P|) .
\end{aligned}
$$

Writing

$$
\zeta_{A}(1+s)=\frac{1}{s \log q}+\frac{1}{2}+\frac{1}{12}(\log q) s+O\left(s^{2}\right)
$$

and

$$
\frac{\zeta_{A}^{\prime}(1+s)}{\zeta_{A}(1+s)}=-s^{-1}+\frac{1}{2} \log q-\frac{1}{12}(\log q)^{2} s+O\left(s^{3}\right)
$$

we have

$$
\begin{aligned}
& \sum_{P \in \mathcal{P}_{2 g+1, q}} \sum_{\gamma_{P}} f\left(\gamma_{P} \frac{2 g \log q}{2 \pi}\right) \\
& =\frac{1}{2 g \log q} \int_{-g}^{g} h(\tau) \sum_{P \in \mathcal{P}_{2 g+1, q}}\left(\log |P|+\frac{\mathcal{X}^{\prime}\left(\frac{1}{2}-\frac{2 \pi i \tau}{2 g \log q}\right)}{\mathcal{X}\left(\frac{1}{2}-\frac{2 \pi i \tau}{2 g \log q}\right)}\right. \\
& \quad+2\left(-\frac{2 g \log q}{4 \pi i \tau}+\frac{1}{2} \log q-\frac{1}{12}(\log q) \frac{4 \pi i \tau}{2 g}-(\log q) e^{(-2 \pi i \tau / 2 g \log q) \log |P|}\right. \\
& \left.\left.\quad \times \mathcal{X}\left(\frac{1}{2}+\frac{2 \pi i \tau}{2 g \log q}\right)\left(-\frac{2 g}{4 \pi i \tau}+\frac{1}{2}-\frac{1}{12} \frac{4 \pi i \tau}{2 g}\right)\right)\right) \mathrm{d} \tau+o(|P|) .
\end{aligned}
$$

then, for $g$ large, only the term $\log |P|$, the $\zeta_{A}^{\prime} / \zeta_{A}$ and the final term in the integral contribute, yielding the asymptotic

$$
\begin{aligned}
& \sum_{P \in \mathcal{P}_{2 g+1, q}} \sum_{\gamma_{P}} f\left(\gamma_{P} \frac{2 g \log q}{2 \pi}\right) \\
& \sim \frac{1}{2 g \log q} \int_{-\infty}^{\infty} h(\tau)\left(\left(\# \mathcal{P}_{2 g+1, q}\right) \log |P|\right. \\
& \left.\quad-\left(\# \mathcal{P}_{2 g+1, q}\right) \frac{2 g \log q}{2 \pi i \tau}+\left(\# \mathcal{P}_{2 g+1, q}\right) e^{-2 \pi i \tau} \frac{2 g \log q}{2 \pi i \tau}\right) \mathrm{d} \tau .
\end{aligned}
$$


However, since $h$ is an even function, we can drop out the middle term and the last term can be duplicated with a change of $\operatorname{sign}$ of $\tau$, leaving

$$
\begin{aligned}
\lim _{g \rightarrow \infty} & \frac{1}{\# \mathcal{P}_{2 g+1, q}} \sum_{P \in \mathcal{P}_{2 g+1, q}} \sum_{\gamma_{P}} f\left(\gamma_{P} \frac{2 g \log q}{2 \pi}\right) \\
& =\int_{-\infty}^{\infty} h(\tau)\left(1+e^{-2 \pi i \tau} \frac{1}{2 \pi i \tau}+e^{2 \pi i \tau} \frac{1}{-2 \pi i \tau}\right) \mathrm{d} \tau \\
& =\int_{-\infty}^{\infty} h(\tau)\left(1+\frac{1}{2 \pi \tau}((\cos (2 \pi \tau)-\sin (2 \pi \tau))-(\cos (2 \pi \tau)-\sin (2 \pi \tau)))\right) \mathrm{d} \tau \\
& =\int_{-\infty}^{\infty} h(\tau)\left(1+\frac{1}{2 \pi \tau}(-2 \sin (2 \pi \tau))\right) d \tau \\
& =\int_{-\infty}^{\infty} h(\tau)\left(1-\frac{\sin (2 \pi \tau)}{\pi \tau}\right) \mathrm{d} \tau .
\end{aligned}
$$

Acknowledgements We would like to express our gratitude to an anonymous referee whose comments improved the presentation of the paper.

Open Access This article is licensed under a Creative Commons Attribution 4.0 International License, which permits use, sharing, adaptation, distribution and reproduction in any medium or format, as long as you give appropriate credit to the original author(s) and the source, provide a link to the Creative Commons licence, and indicate if changes were made. The images or other third party material in this article are included in the article's Creative Commons licence, unless indicated otherwise in a credit line to the material. If material is not included in the article's Creative Commons licence and your intended use is not permitted by statutory regulation or exceeds the permitted use, you will need to obtain permission directly from the copyright holder. To view a copy of this licence, visit http://creativecommons.org/licenses/by/4.0/.

\section{References}

1. Andrade, J.C.: Random matrix theory and $L$-functions in Function Fields, PhD Thesis, University of Bristol (2012)

2. Andrade, J.C., Keating, J.P.: The mean value of $L\left(\frac{1}{2}, \chi\right)$ in the hyperelliptic ensemble. J. Number Theory 132, 2793-2816 (2012)

3. Andrade, J.C., Keating, J.P.: Mean value theorem for $L$-functions over prime polynomials for the rational function field. Acta Arith. 161, 371-385 (2013)

4. Andrade, J.C., Keating, J.P.: Conjectures for the integral moments and ratios of $L$-functions over function fields. J. Number Theory 142, 102-148 (2014)

5. Baluyot, S., Pratt, K.: Dirichlet L-functions of quadratic characters of prime conductor at the central point. arXiv:1809.09992 (2018)

6. Bui, H., Florea, A.: Moments of Dirichlet $L$-functions with prime conductors over function fields. preprint arXiv:1909.00854 (2019)

7. Conrey, J.B., Ghosh, A.: Mean values of the Riemann zeta-function III, Proceedings of the Amalfi Conference on Analytic Number Theory, Universitá di Salerno, (1992)

8. Conrey, J.B., Gonek, S.M.: High moments of the Riemann zeta-function. Duke Math. J. 107(3), 577604 (2001)

9. Conrey, J.B., Keating, J.P.: Moments of zeta and correlations of divisor-sums: II. In: Alaca, A., Alaca, S., Williams, K.S. (eds.) Advances in the Theory of Numbers, pp. 75-85. Springer, New York (2015)

10. Conrey, J.B., Keating, J.P.: Moments of zeta and correlations of divisor-sums: III. Indag. Math. 26, 736-747 (2015) 
11. Conrey, J.B., Keating, J.P.: Moments of zeta and correlations of divisor-sums: I. Philos. Trans. R. Soc. A 373, 20140313 (2015)

12. Conrey, J.B., Keating, J.P.: Moments of zeta and correlations of divisor-sums: IV. Res. Number Theory 1, 1-24 (2016)

13. Conrey, J.B., Snaith, N.C.: Applications of the $L$-functions ratios conjectures. Proc. Lond. Math. Soc. 3(94), 594-646 (2007)

14. Conrey, J.B., Farmer, D.W., Keating, J.P., Rubinstein, M.O., Snaith, N.C.: Integral moments of $L-$ functions. Proc. Lond. Math. Soc. 91, 33-104 (2005)

15. Conrey, J.B., Farmer, D.W., Zirnbauer, M.R.: Autocorrelation of ratios of $L$-functions. Commun. Number Theory Phys. 2(3), 593-636 (2008)

16. Diaconu, A., Whitehead, I.: On the third moment of $L\left(\frac{1}{2}, \chi_{d}\right)$ II: the number field case. arXiv:1804.00690 (2018)

17. Diaconu, A., Goldfeld, D., Hoffstein, J.: Multiple Dirichlet series and moments of zeta and $L$-functions. Compos. Math. 139, 297-360 (2003)

18. Florea, A.: Improving the error term in the mean value of $L(1 / 2, \chi)$ in the hyperelliptic ensemble. Int. Math. Res. Notices 20, 6119-6148 (2016)

19. Florea, A.: The second and the third moment of $L\left(\frac{1}{2}, D\right)$ in the hyperelliptic ensemble. Forum Math. 29(4), 873-892 (2017)

20. Florea, A.: The fourth moment of $L(1 / 2, \chi)$ in the hyperelliptic ensemble. GAFA 27, 541-595 (2017)

21. Goldfeld, D., Viola, C.: Mean values of $L$-functions associated to elliptic, Fermat and other curves at the centre of the critical strip. J. Number Theory 11, 305-320 (1979)

22. Hardy, G.H., Littlewood, J.E.: Contributions to the theory of the Riemann zeta-function and the theory of the distribution of primes. Acta Math. 41, 119-196 (1918)

23. Hoffstein, J., Rosen, M.: Average values of $L$-series in function fields. J. Reine Angew. Math. 426, 117-150 (1992)

24. Ingham, A.E.: Mean-value theorems in the theory of the Riemann zeta-function. Proc. Lond. Math. Soc. 27, 273-300 (1926)

25. Jutila, M.: On the mean value of $L(1 / 2$, ) for real characters. Analysis 1, 149-161 (1981)

26. Keating, J.P., Odgers, B.E.: Symmetry transitions in random matrix theory and $L$-functions. Commun. Math. Phys. 281, 499-528 (2008)

27. Keating, J.P., Snaith, N.C.: Random matrix theory and $L$-functions at $s=1 / 2$. Commun. Math. Phys. 214(1), 91-110 (2000)

28. Keating, J.P., Snaith, N.C.: Random matrix theory and $\zeta(1 / 2+i t)$. Commun. Math. Phys. 214(1), 57-89 (2000)

29. Rosen, M.: Number theory in function fields. Graduate Text in Mathematics vol.210. Springer-Verlag, New York (2002)

30. Rudnick, Z.: Traces of high power of the Frobenius class in hyperelliptic ensemble. Acta Arith. 143, 81-99 (2010)

31. Shen, Q: The fourth moment of quadratic Dirichlet $L$-functions. arXiv:1907.01107 (2019)

32. Soundararajan, K.: Nonvanishing of quadratic Dirichlet $L$-functions at $s=1$. Ann. Math. 152(2), 447-488 (2000)

33. Soundararajan, K., Young, M.P.: The second moment of quadratic twists of modular $L$-functions. J. Eur. Math. Soc. 12(5), 1097-1116 (2010)

Publisher's Note Springer Nature remains neutral with regard to jurisdictional claims in published maps and institutional affiliations. 